\title{
Manifolds of Positive Scalar Curvature: a Progress Report
}

\author{
Jonathan Rosenberg
}

The scalar curvature $\kappa$ is the weakest curvature invariant one can attach (pointwise) to a Riemannian $n$-manifold $M^{n}$. Its value at any point can be described in several different ways:

(1) as the trace of the Ricci tensor, evaluated at that point.

(2) as twice the sum of the sectional curvatures over all 2-planes $e_{i} \wedge e_{j}$, $i<j$, in the tangent space to the point, where $e_{1}, \ldots, e_{n}$ is an orthonormal basis.

(3) up to a positive constant depending only on $n$, as the leading coefficient in an expansion [22, Theorem 3.1]

$$
V_{M}(r)=V_{E}(r)\left(1-\frac{\kappa}{6(n+2)} r^{2}+\cdots\right)
$$

telling how the volume $V_{M}(r)$ of a small geodesic ball in $M$ of radius $r$ differs from volume $V_{E}(r)=C_{n} r^{n}$ of a corresponding ball in Euclidean space. Positive scalar curvature means balls of radius $r$ for small $r$ have a smaller volume than balls of the same radius in Euclidean space; negative scalar curvature means they have larger volume.

In the special case $n=2$, the scalar curvature is just twice the Gaussian curvature.

This paper will deal with bounds on the scalar curvature, and especially, with the question of when a given manifold (always assumed $C^{\infty}$ ) admits a Riemannian metric with positive or non-negative scalar curvature. (If the manifold is non-compact, we require the metric to be complete; otherwise this is no restriction at all.) We will not go over the historical development of this subject or everything that is known about it; instead, our focus here will be on updating the existing surveys $[\mathbf{2 0}],[\mathbf{6 8}],[6 \mathbf{6 9}]$ and $[\mathbf{5 8}]$.

This work was partially supported by NSF grant number DMS-0504212. 
We should explain why we care so much about positivity of the scalar curvature. Why not ask about metrics of negative scalar curvature, or of vanishing scalar curvature, or of non-negative scalar curvature? More generally, we could ask which smooth functions on a closed manifold $M$ are realized as the scalar curvature function of some metric on $M$. It is a remarkable result of Kazdan and Warner that (in dimensions >2) the answer to this question only depends on which of the following classes the manifold $M$ belongs to:

(1) Closed manifolds admitting a Riemannian metric whose scalar curvature function is non-negative and not identically 0 .

(2) Closed manifolds admitting a Riemannian metric with vanishing scalar curvature, and not in class (1).

(3) Closed manifolds not in classes (1) or (2).

All these three classes are non-empty if $n \geq 2$. By a simple application of the Gauss-Bonnet Theorem, if $n=2$, class (1) consists of $S^{2}$ and $\mathbb{R P}^{2}$; class (2) consists of $T^{2}$ and the Klein bottle; and class (3) consists of surfaces with negative Euler characteristic.

Theorem 0.1 ("Trichotomy Theorem" [34], [33], [32]). Let $M^{n}$ be a closed connected manifold of dimension $n \geq 3$.

(1) If $M$ belongs to class (1), every smooth function is realized as the scalar curvature function of some Riemannian metric on $M$.

(2) If $M$ belongs to class (2), then a function $f$ is the scalar curvature of some metric if and only if either $f(x)<0$ for some point $x \in M$, or else $f \equiv 0$. If the scalar curvature of some metric $g$ vanishes identically, then $g$ is Ricci flat (i.e., not only does the scalar curvature vanish identically, but so does the Ricci tensor).

(3) If $M$ belongs to class (3), then $f \in C^{\infty}(M)$ is the scalar curvature of some metric if and only if $f(x)<0$ for some point $x \in M$.

This Theorem thus shows that deciding whether a manifold $M$ belong to class (1) is equivalent to determining whether $M$ admits a metric of strictly positive scalar curvature. Furthermore, in this case, there are no restrictions at all on possibilities for the scalar curvature. We will include some more results about class (2) in the last section of this paper.

REMARK 0.2. Note that Theorem 0.1 partially justifies the comment above, that existence of a metric of positive scalar curvature on a noncompact (connected) manifold $M$ is no restriction at all if the metric is not required to be complete. Indeed, suppose $M$ is diffeomorphic to an open subset of a compact manifold with boundary $\bar{M}$. (If $M$ is homotopically finite with "tame" ends, this is not much of a restriction.) Take the double of $\bar{M}$ along $\partial \bar{M}$; this is now a closed manifold $X$ in which $M$ is embedded as an open subset with complement having non-empty interior. By Theorem 0.1 , there is a metric on $X$ whose scalar curvature function is positive on $M$ but negative somewhere in the complement of $M$. The general case (where 
$M$ cannot be embedded in a closed manifold) can be deduced from this case with somewhat more work.

Most of the results presented in this paper are due to other authors, but the organization here may be a bit different than in the original sources. In Section 1, we will discuss necessary and sufficient conditions for a closed manifold $M$ to admit a metric of positive scalar curvature. Part of this discussion (e.g., Conjecture 1.19, Theorem 1.20, and Remark 1.25) has been known for a while to the experts but may not be in the literature in its present form. In Section 2, we will discuss the topology of the space of metrics of positive scalar curvature in cases where this space is non-empty. Some of the proofs in this section are new. Then Section 3 will discuss the question of what non-compact manifolds admit a complete metric of positive scalar curvature. Here, Theorem 3.4, Corollary 3.5, and part of Theorem 3.9 are new results. Section 4 will discuss a few other miscellaneous topics.

\section{The obstruction problem: which closed manifolds admit a metric of positive scalar curvature?}

If $M^{n}$ is a closed $n$-manifold, when can $M$ be given a Riemannian metric for which the scalar curvature function is everywhere strictly positive? (For simplicity, such a metric will henceforth be called a metric of positive scalar curvature.)

Answering this basic question involves two disjoint sets of techniques: obstruction results, showing that some manifolds do not admit metrics of positive scalar curvature, and positive results, showing that many manifolds do admit such metrics.

1.1. Obstruction results. All known obstruction results follow from one of three basic principles:

(1) The result of Lichnerowicz [40], that if $\not D$ is the Dirac operator on a spin manifold $M$ (a self-adjoint elliptic first-order differential operator, acting on sections of the spinor bundle), then

$$
\not D^{2}=\nabla^{*} \nabla+\frac{\kappa}{4}
$$

Here $\nabla$ is the covariant derivative on the spinor bundle induced by the Levi-Civita connection, and $\nabla^{*}$ is the adjoint of $\nabla$. Since the operator $\nabla^{*} \nabla$ is obviously self-adjoint and non-negative, it follows from equation (1.1) that the square of the Dirac operator for a metric of positive scalar curvature is bounded away from 0 , and thus that the Dirac operator cannot have any kernel. It follows that any index-like invariant of $M$ which can be computed in terms of harmonic spinors (i.e., the kernel of $\not D$ ) has to vanish. 
(2) The Schoen-Yau minimal surface technique [61], which implies that if $M^{n}$ is an oriented manifold of positive scalar curvature, and if $N^{n-1}$ is a closed stable minimal hypersurface in $M$ dual to a nonzero class in $H^{1}(M, \mathbb{Z})$, then $N$ also admits a metric of positive scalar curvature.

(3) The Seiberg-Witten technique [73], which implies that if $M^{4}$ is a closed 4-manifold with a non-zero Seiberg-Witten invariant, then $M$ does not admit a metric of positive scalar curvature.

Each of these three techniques has its own advantages and disadvantages. Technique (1) applies to manifolds of all dimensions, and is usually the most powerful, but it only applies to spin manifolds, or at least to manifolds with a spin cover (i.e., to manifolds $M$ such that $w_{2}(\widetilde{M})=0$, where $\widetilde{M}$ is the universal cover of $M$ and $w_{2}$ is the second Stiefel-Whitney class). Technique (2) applies whether or not $M$ and $N$ are spin, but it requires $H^{1}(M, \mathbb{Z})$ to be non-zero, which is quite a restriction on $\pi_{1}(M)$. In addition, since solutions to the minimal hypersurface equations in general have singularities, this technique only works without modification up to dimension 7 or 8 . There have been hopes for a long time (see for example [64]) that one could "excise the singularities" to make this technique work in high dimensions, and now Lohkamp [43] has announced a precise result of this sort, based in part on joint work with Ulrich Christ, though as of the time of writing this paper, the details have not yet appeared. Finally, technique (3) again does not require a spin condition, but works only in the special dimension 4. (Sometimes one can reduce problems about manifolds in dimensions 5 through 8 to this case using technique (2).) Let's now go into the three techniques in somewhat more detail.

1.1.1. The Dirac obstruction. We start with (1) of $\S 1.1$, the Dirac operator method. If $M$ is a spin manifold of dimension $n$, there is a version of the Dirac operator which commutes with the action of the Clifford algebra $C \ell_{n}$ (see $[37, \S I I .7])$. In particular, its kernel is a (graded) $C \ell_{n}$-module, which represents an element $\alpha(M)$ in the real $K$-theory group $K O_{n}=K O^{-n}(\mathrm{pt})$ (see [37, Def. II.7.4]).

Theorem 1.1 (Lichnerowicz [40]; Hitchin [29]). If $M^{n}$ is a closed spin manifold for which $\alpha(M) \neq 0$ in $K O_{n}$, then $M$ does not admit a metric of positive scalar curvature.

We recall that $K O_{n} \cong \mathbb{Z}$ for $n \equiv 0 \bmod 4$, that $K O_{n} \cong \mathbb{Z} / 2$ for $n \equiv 1,2$ $\bmod 8$, and $K O_{n}=0$ for all other values of $n$. Furthermore, for $n \equiv 0 \bmod$ 4, the invariant $\alpha(M)$ is essentially equal to Hirzebruch's $\widehat{A}$-genus $\widehat{A}(M)$, namely $\alpha(M)=\widehat{A}(M)$ for $n \equiv 0 \bmod 8$, and $\alpha(M)=\widehat{A}(M) / 2$ for $n \equiv 4$ mod 8 . So this result immediately shows that there are many manifolds, even simply connected ones, which do not lie in class (1) of the KazdanWarner trichotomy (see Theorem 0.1). E.g., the Kummer surface $K^{4}$, the hyperplane in the complex projective space $\mathbb{C P}^{3}$ given by the equation 
$z_{0}^{4}+z_{1}^{4}+z_{2}^{4}+z_{3}^{4}=0$, is spin and has $\widehat{A}(K)=2$, and hence does not admit a metric of positive scalar curvature.

We observe that $\alpha(M)$ depends only on the spin bordism class $[M] \in$ $\Omega_{n}^{\text {spin }}$. In fact, we can interpret $\alpha(M)$ as the image of $[M]$ under a natural transformation of generalized homology theories as follows. Let $K O_{*}(X)$ and $k o_{*}(X)$ denote the periodic and connective real $K$-homology of a space $X$, respectively (so $K O_{*}(X)$ satisfies Bott periodicity, and the spectrum defining $k o_{*}$ is obtained from the periodic $K O$-spectrum by killing all homotopy groups in negative degrees). Then there are natural transformations

$$
\Omega_{*}^{\text {spin }}(X) \stackrel{D}{\longrightarrow} k O_{*}(X) \stackrel{\text { per }}{\longrightarrow} K O_{*}(X),
$$

the first of which sends the bordism class $[M, f]$ to $f_{*}\left([M]_{k o}\right)$, where $[M]_{k o} \in$ $k o_{*}(M)$ denotes the $k o$-fundamental class of $M$ determined by the spin structure, and the second of which builds in Bott periodicity by inverting the Bott generator of $k o_{8} \cong \mathbb{Z}$. With this notation, $\alpha(M)=$ per $\circ D([M])$ (in the case $X=\mathrm{pt})$.

A stronger result than Theorem 1.1 can be obtained by taking the fundamental group into account and coupling the Dirac operator with flat or almost flat vector bundles. To get good results, we need to use infinitedimensional bundles, or at least sequences of bundles whose dimensions go to infinity. Here we will use the index theory of Mishchenko and Fomenko [46] and bundles of Hilbert $C^{*}$-modules over the real $C^{*}$-algebra of the fundamental group. This algebra, denoted $C_{\mathbb{R}}^{*}(\pi)$, is the completion of the group ring $\mathbb{R}[\pi]$ for the largest $C^{*}$-norm, or in other words the largest operator norm on a Hilbert space, when one lets $\mathbb{R}[\pi]$ act on Hilbert spaces via representations of $\pi$ by invertible isometries. Ordinary flat vector bundles can't give very much, since the rational characteristic classes of any finitedimensional flat vector bundle are trivial by Chern-Weil theory.

We will also need a topological construction that will play a big role later. For any (discrete) group $\pi$, there is a classifying space $B \pi$, which we can choose to be a CW complex, having $\pi$ as fundamental group and with contractible universal cover $E \pi$. This space is unique up to homotopy equivalence. If $M$ has fundamental group $\pi$, then there is a classifying map $f: M \rightarrow B \pi$ which induces an isomorphism on fundamental groups. This map is determined up to homotopy by an identification of $\pi_{1}(M)$ with $\pi$. Thus we can replace $X$ by $B \pi$ in (1.2) above and define, if $M^{n}$ is a spin manifold, an invariant $\alpha_{B \pi}(M) \in K O_{n}(B \pi)$.

The best result one can obtain on the obstruction problem using the index theory of the Dirac operator can be stated in the case of spin manifolds as follows:

ThEOREM 1.2 (Rosenberg [54]). For any discrete group $\pi$, there is a natural assembly map $A: K O_{*}(B \pi) \rightarrow K O_{*}\left(C_{\mathbb{R}}^{*}(\pi)\right)$ from the KO-homology of the classifying space to the topological $K$-theory of the real group $C^{*}$ algebra. (The Baum-Connes Conjecture implies, in particular, that this map 
is injective if $\pi$ is torsion-free.) If $M^{n}$ is a closed spin manifold for which $A\left(\alpha_{B \pi}(M)\right) \neq 0$ in $K O_{n}\left(C_{\mathbb{R}}^{*}(\pi)\right)$, then $M$ does not admit a metric of positive scalar curvature.

Sketch. Form the bundle $\mathcal{V}_{B \pi}=E \pi \times_{\pi} C_{\mathbb{R}}^{*}(\pi)$ over $B \pi$ whose fibers are rank-one free (right) modules over $C_{\mathbb{R}}^{*}(\pi)$. As a " $C_{\mathbb{R}}^{*}(\pi)$-vector bundle" over $B \pi$, this has a stable class $\left[\mathcal{V}_{B \pi}\right]$ in a $K$-group $K O^{0}\left(B \pi ; C_{\mathbb{R}}^{*}(\pi)\right)$, and $A$ is basically the "slant product" with $\left[\mathcal{V}_{B \pi}\right]$. This relies on an index theory, due to Mishchenko and Fomenko, for elliptic operators with coefficients in a $C_{\mathbb{R}}^{*}(\pi)$-vector bundle. If $M$ is as in the theorem, then the (Clifford algebra linear) Dirac operator on $M$, with coefficients in the bundle $\mathcal{V}_{B \pi}$, has an index $\alpha_{B \pi}(M, f) \in K O_{n}\left(C_{\mathbb{R}}^{*}(\pi)\right)$, which one can show by the Kasparov calculus is just $A \circ$ pero $D([M, f])$. Since $\mathcal{V}_{B \pi}$ is by construction a flat bundle, there are no correction terms due to curvature of the bundle, and formula (1.1) applies without change. Hence if $M$ has positive scalar curvature, the square of this Dirac operator is bounded away from 0 , and the index vanishes.

REMARK 1.3. For purposes of the construction above, one could just as well use the reduced real group $C^{*}$-algebra $C_{\mathbb{R}, \text { red }}^{*}(\pi)$, which is the completion of $\mathbb{R}[\pi]$ for its action on $L^{2}(\pi)$ by left convolution. When $\pi$ is amenable, this algebra coincides with $C_{\mathbb{R}}^{*}(\pi)$; otherwise, it is a proper quotient. The assembly map into $K O_{*}\left(C_{\mathbb{R}}^{*}(\pi)\right)$ potentially has a smaller kernel than the one into $K O_{*}\left(C_{\mathbb{R} \text {,red }}^{*}(\pi)\right)$, but on the other hand, the latter has a better chance of being an isomorphism. The Baum-Connes Conjecture would imply that if $\pi$ is torsion-free, the assembly map into $K O_{*}\left(C_{\mathbb{R} \text {,red }}^{*}(\pi)\right)$ is an isomorphism, whereas one cannot generally expect this for the assembly map into $K O_{*}\left(C_{\mathbb{R}}^{*}(\pi)\right)$.

It was conjectured in [55], admittedly on the basis of rather flimsy evidence, that when $\pi$ is finite and $M^{n}$ is a spin manifold, the vanishing of $A\left(\alpha_{B \pi}(M)\right)$ is not only necessary, but also sufficient, for $M$ to admit a metric of positive scalar curvature. This conjecture is usually called the GromovLawson-Rosenberg Conjecture. (See Conjecture 1.22 below.) There are no known counterexamples to this conjecture in dimensions $n \geq 5$, though as we will see, there are reasons to be skeptical about it.

There is an analogue of Theorem 1.2 that holds when $M$ does not admit a spin structure, but $w_{2}(\widetilde{M})=0$. We call this the "twisted" case. The statement appears a bit technical, and those not so interested in having the most general possible result can ignore it and concentrate on just two cases: the case where $M$ is spin, which we have already discussed, and the case where $M$ is oriented and $w_{2}(\widetilde{M}) \neq 0$, in which case the Dirac operator method gives no information at all.

The following definitions are due to Stolz. 
Definition 1.4. Let $\gamma$ be a triple $(\pi, w, \widehat{\pi})$, where $w: \pi \rightarrow \mathbb{Z} / 2$ is a group homomorphism (this will correspond to $w_{1}$ of our manifold) and $\widehat{\pi} \rightarrow \pi$ is an extension of $\pi$ such that $\operatorname{ker}(\widehat{\pi} \rightarrow \pi)$ is either $\mathbb{Z} / 2$ or the trivial group. Let $\sigma: \operatorname{Spin}(n) \rightarrow S O(n)$ be the non-trivial double covering of the special orthogonal group $S O(n)$. We note that the conjugation action of $O(n)$ on $S O(n)$ lifts to an action on $\operatorname{Spin}(n)$. Let $\widehat{\pi} \ltimes \operatorname{Spin}(n)$ be the semi direct product, where $\widehat{g} \in \widehat{\pi}$ acts on the normal subgroup $\operatorname{Spin}(n)$ by conjugation by $r^{w(\widehat{g})}$. Here $r \in O(n)$ is the reflection in the hyperplane perpendicular to $e_{1}=(1,0, \ldots, 0) \in \mathbb{R}^{n}$. Abusing notation, we also use the notation $w$ for the composition $\widehat{\pi} \rightarrow \pi \rightarrow \mathbb{Z} / 2$. We define $G(\gamma, n)$ to be the quotient of $\widehat{\pi} \ltimes \operatorname{Spin}(n)$ by the central subgroup generated by $(k,-1)$, where $k \in \widehat{\pi}$ is the (possibly trivial) generator of $\operatorname{ker}(\widehat{\pi} \rightarrow \pi)$. Sending $[a, b] \in G(\gamma, n)$ to $r^{w(a)} \sigma(b)$ defines a homomorphism $\rho(\gamma, n): G(\gamma, n) \rightarrow O(n)$.

A $\gamma$-structure on an $n$-dimensional Riemannian manifold $M$ is a principal $G(\gamma, n)$-bundle $P \rightarrow M$ together with a $G(\gamma, n)$-equivariant map $\rho: P \rightarrow$ $O(M)$. Here $O(M)$ is the orthogonal frame bundle of $M$, a principal bundle for the orthogonal group $O(n)$, and $G(\gamma, n)$ acts on $O(M)$ via the homomorphism $\rho(\gamma, n)$.

REMARK 1.5. Let $M$ be a connected manifold with fundamental group $\pi$ and with $w_{1}(M)=w: \pi \rightarrow \mathbb{Z} / 2$. Then $M$ always admits a $\gamma$-structure for some $\gamma=(\pi, w, \widehat{\pi})$. We can arrange to have $\operatorname{ker}(\widehat{\pi} \rightarrow \pi) \neq 0$ exactly when $w_{2}(\widetilde{M})=0$.

(1) If $\pi$ is the trivial group, then $G(\gamma, n)=S O(n)$ (resp. Spin $(n))$ if $\operatorname{ker}(\widehat{\pi} \rightarrow \pi)$ is trivial (resp. non-trivial). In this case a $\gamma$-structure on $M$ amounts to an orientation (resp. spin structure) on $M$ (cf. [37, Def. II.1.3]).

(2) More generally, if $w=0$ and $\widehat{\pi}=\pi$ (resp. $\widehat{\pi}=\pi \times \mathbb{Z} / 2$ ), then $G(\gamma, n)=\pi \times S O(n)($ resp. $G(\gamma, n)=\pi \times \operatorname{Spin}(n))$; in this case, a $\gamma$-structure amounts to an orientation (resp. spin structure) on $M$, together with a principal $\pi$-bundle $\widetilde{M} \rightarrow M$.

(3) If $M$ is not orientable, so $w \neq 0$, but $w_{2}(\widetilde{M}) \neq 0$, then $G(\gamma, n)=$ $\pi \times O(n)$ and the $\gamma$-structure on $M$ is determined by the classifying map $M \rightarrow B \pi \times B O(n)$, where the first component of the map is the classifying map for the universal covering, and the second component is the classifying map for the tangent bundle.

(4) A $\gamma$-structure determines a principal $\pi$-bundle $\widetilde{M} \stackrel{\text { def }}{=} P / G_{1} \rightarrow M$, where $G_{1}$ is the identity component of $G(\gamma, n)$. We note that $G_{1}=$ $S O(n)$ if $\operatorname{ker}(\widehat{\pi} \rightarrow \pi)$ is trivial, and $G_{1}=\operatorname{Spin}(n)$ otherwise. Hence the principal $G_{1}$-bundle $P \rightarrow \widetilde{M}$ can be identified with the oriented frame bundle of $\widetilde{M}$ or a double cover thereof.

The substitute for Theorem 1.2 in the twisted case involves a substitute $C^{*}(\gamma)$ for $C_{\mathbb{R}}^{*}(\pi)$. This is defined as follows. 
Definition 1.6. Let $\gamma=(\pi, w, \widehat{\pi})$ be a $\gamma$-structure as in Definition 1.4. We define a $\mathbb{Z} / 2$-graded $C^{*}$-algebra $C^{*} \gamma$ to be the -1-eigenspace for the involution on the group $C^{*}$-algebra $C^{*} \widehat{\pi}$ defined by multiplication by the central generator $k$ of $\operatorname{ker}(\widehat{\pi} \rightarrow \pi)$. The $\mathbb{Z} / 2$-grading is given by the $\{ \pm 1\}$ eigenspaces of the involution $C^{*} \gamma \rightarrow C^{*} \gamma$ which is the restriction of the involution $C^{*} \widehat{\pi} \rightarrow C^{*} \widehat{\pi}$ given by $\hat{g} \mapsto(-1)^{\hat{w}(\hat{g})}$ for $\hat{g} \in \widehat{\pi} \subset C^{*} \widehat{\pi}$, where $\hat{w}$ is the composition of the projection map $\hat{\pi} \rightarrow \pi$ and $w: \pi \rightarrow \mathbb{Z} / 2$. In particular, $C^{*} \gamma=0$ if $\widehat{\pi}=\pi$ and $C^{*} \gamma=C^{*} \pi$ (with the trivial grading) if $w=0$ and $\widehat{\pi}=\pi \times \mathbb{Z} / 2$.

The analogue of Theorem 1.2 in the twisted case is then:

TheOREM 1.7 (Stolz; see [58, §5]). If $M^{n}$ is a closed manifold with $w_{2}(\widetilde{M})=0$ and with $\gamma$-structure $\gamma=(\pi, w, \widehat{\pi})$, then there is a "twisted Dirac obstruction" in $\mathrm{KO}_{n}\left(C^{*} \gamma\right)$, whose vanishing is necessary for $M$ to admit a metric of positive scalar curvature.

Roughly speaking, this theorem, like Theorem 1.2, is proved by taking the "index of the Dirac operator" in a suitable sense. Since our understanding of positive scalar curvature is incomplete enough even for spin manifolds, or for oriented manifolds with non-spin universal cover, we will concentrate hereafter on these simpler cases and not mention the twisted case any further.

1.1.2. The minimal hypersurface method. Now let's discuss (2) of $\S 1.1$, the minimal hypersurface method. This relies on the following inequality, found in $[\mathbf{6 1}]$ :

Lemma 1.8 (Schoen-Yau [61]). Let $M^{n}$ be a closed oriented n-manifold with $H^{1}(M, \mathbb{Z}) \neq 0$ and with positive scalar curvature, and let $H^{n-1}$ be a stable minimal hypersurface, minimizing $(n-1)$-dimensional volume in its homology class. Then

$$
\int_{H}\left(\frac{\kappa \phi^{2}}{2}+|\nabla \phi|^{2}\right) d \mathrm{vol}>0,
$$

with $\kappa$ the scalar curvature of $H$ in the induced metric from $M$ and $d$ vol the measure on $H$ defined by the induced metric, for all functions $\phi \in C^{\infty}(H)$ not vanishing identically. (Here $\nabla$ is to be computed with respect to the induced metric on $\mathrm{H}$.)

From this one can deduce that $H$ also has a metric of positive scalar curvature, and in some cases, this leads to a contradiction, with the result that $M$ could not have had a metric of positive scalar curvature in the first place. For example, if $n=3$, then taking $\phi \equiv 1$ in (1.3), we deduce that the integral of $\kappa$ over $H$ is positive, which by Gauss-Bonnet implies that $H$ must be a sphere. In particular, since the homology class of $H$ in $H_{2}(M, \mathbb{Z})$ is represented by a sphere, it lies in the image of the Hurewicz map $\pi_{2}(M) \rightarrow$ 
$H_{2}(M, \mathbb{Z})$. This is impossible if $M$ is aspherical with $b_{1}(M)>0$ (so that we could construct $H$ in the first place), so we see that an aspherical oriented closed 3-manifold $M$ with $b_{1}(M)>0$ cannot have a metric of positive scalar curvature. This sort of reasoning was refined in [62] to show that if $M$ is a compact oriented closed 3-manifold $M$ with $\pi_{1}(M)$ containing a product of two cyclic groups or a subgroup isomorphic to the fundamental group of a compact Riemann surface of genus $>1$, then $\mathrm{M}$ cannot admit a metric of positive scalar curvature.

If $n>3$, the reasoning to get from (1.3) to the fact that $H$ admits a metric of positive scalar curvature is a bit more complicated. Basically, (1.3) implies that the conformal Laplacian of $H$ (for the metric induced from $M$ ) is strictly positive, which in turn implies (by an argument of Kazdan and Warner) that one can make a conformal change in the metric of $H$ to achieve positive scalar curvature.

Iterating use of this technique, one can show that many manifolds do not admit metrics of positive scalar curvature, as long as one can produce chains of stable minimal hypersurfaces going down in dimension from $n$ to 2. For example, one can formalize this as follows:

TheOREM 1.9 (Schick, [60, Corollary 1.5]). Let $X$ be any space, let $3 \leq k \leq 8$, and let $\alpha \in H^{1}(X, \mathbb{Z})$. Let $H_{k}(X, \mathbb{Z})^{+}$denote the subset of $H_{k}(X, \mathbb{Z})$ consisting of classes $f_{*}([N])$, where $f: N^{k} \rightarrow X$ and $N$ is an oriented closed manifold of positive scalar curvature. Then cap product with $\alpha$ maps $H_{k}(X, \mathbb{Z})^{+}$to $H_{k-1}(X, \mathbb{Z})^{+}$.

Here the restriction to $k \leq 8$ is simply for the purpose of knowing that if $f: N^{k} \rightarrow X$, then there is a smooth, nonsingular minimal hypersurface of $N$ dual to $f^{*} \alpha$. Schick's original paper had $k \leq 7$; this can be improved, as remarked in $[\mathbf{3 1}]$, using better regularity results for minimal hypersurfaces in [65]. Presumably the dimensional restriction can be removed altogether using the results of $[\mathbf{4 3}]$.

The minimal hypersurface technique is especially powerful in low dimensions. For example, it was used to prove:

Theorem 1.10 (Schoen and Yau [64, Theorem 6]). No closed aspherical 4-manifold can admit a metric of positive scalar curvature.

1.1.3. The Seiberg-Witten method. Finally we get to (3) of $\S 1.1$, the Seiberg-Witten method. This applies only to oriented closed 4-manifolds. Any such manifold $M$ always admits a $\operatorname{spin}^{c}$ structure $\xi$. It is not unique (in fact, the set of $\operatorname{spin}^{c}$ structures compatible with the orientation is a principal homogeneous space for $H^{2}(M, \mathbb{Z})$ ), but for each choice, provided $b_{2}^{+}(M)>1$, there is an integer invariant, called the Seiberg-Witten invariant $\mathrm{SW}(\xi)$, which counts the number of solutions to a certain non-linear elliptic system of partial differential equations. (The equations concern a spinor field $\psi$, i.e., 
a section of the positive half-spinor bundle $\mathcal{S}_{\xi}^{+}$associated to $\xi$, plus a connection $A$ on the line bundle $\bigwedge^{2} \mathcal{S}_{\xi}^{+}$. We require $\psi$ to satisfy the Dirac equation defined by the connection, $D_{\xi, A}(\psi)=0$, and in the "unperturbed" version of the equations, ${ }^{1}$ also require the self-dual part of the curvature of $A$ to be given by the pairing of $\psi$ with itself under the nontrivial bundle map

$$
\mathcal{S}_{\xi}^{+} \otimes \mathcal{S}_{\xi}^{+} \rightarrow \Omega_{+}^{2}
$$

which of course is quadratic, not linear, in $\psi$.) When $b_{2}^{+}(M)=1$, it is still possible to define $\mathrm{SW}(\xi)$, but in general it also depends on the Riemannian metric (or the perturbation made to the equation). The basic connection between Seiberg-Witten invariants and scalar curvature is the following:

Theorem 1.11 (Witten [73], [47, Corollary, 5.1.8]). Let $M$ be an oriented closed 4-manifold with $b_{2}^{+}(M)>1$. If $\mathrm{SW}(\xi) \neq 0$ for some spin ${ }^{c}$ structure $\xi$, then $M$ does not admit a metric of positive scalar curvature.

On the other hand, for some special classes of 4-manifolds, one knows that the Seiberg-Witten invariant can be non-zero. For example, one has:

TheOREM 1.12 (Taubes [70]). Let $M^{n}$ be a closed, connected oriented 4manifold with $b_{2}^{+}(M)>1$. If $M$ admits a symplectic structure (in particular, if $M$ admits the structure of a Kähler manifold of complex dimension 2), then $\mathrm{SW}(\xi) \neq 0$ for some spin ${ }^{c}$ structure $\xi$, and thus $M$ does not admit a positive scalar curvature metric (even one not well-behaved with respect to the symplectic structure).

This dramatic result implies that many smooth 4-manifolds do not admit metrics of positive scalar curvature, even if they are non-spin and simply connected. In other words:

Counterexample 1.13. In dimension 4, there exist:

(1) a simply connected spin manifold $M^{4}$ with $\widehat{A}(M)=0$ but with no positive scalar curvature metric.

(2) simply connected non-spin manifolds with no positive scalar curvature metric.

Still more subtle things go wrong in dimension 4, such as:

Counterexample 1.14 (Hanke, Kotschick, and Wehrheim [25]). For any odd prime $p$, there exists a smooth spin 4-manifold $M^{4}$ with fundamental group cyclic of order $p$, such that $M$ does not admit a metric of positive scalar curvature, but its universal cover does.

\footnotetext{
${ }^{1}$ Sometimes, for technical reasons, one needs to make a small perturbation. See $[\mathbf{4 7}$, Chapter 6] for a detailed explanation. We'll ignore this for the moment.
} 
When $b_{2}^{+}(M)=1$, then Seiberg-Witten invariants still exist, but they are not necessarily independent of the metric $g$. However, it is still true that if $\mathrm{SW}(\xi, g) \neq 0$, then the metric $g$ cannot have positive scalar curvature. This is useful in some cases, since for example, one can show that if $M$ is homeomorphic to $\mathbb{C P}^{2} \# n \overline{\mathbb{C P}^{2}}$ with $n \leq 9$, then the Seiberg-Witten invariants are actually independent of the choice of metric. This was used in [48], [66], [15], and [49] to construct exotic 4-manifolds, homeomorphic to classical simply connected manifolds of positive scalar curvature, without metrics of positive scalar curvature. We will see that all of this is very different from what happens in dimensions $\geq 5$.

1.2. Positive results. The known positive results about existence of metrics of positive scalar curvature come from a combination of

(1) specific constructions for certain special classes of manifolds, such as fiber bundles with fibers of positive scalar curvature and the structure group consisting of isometries (see, e.g., [67, Observation, p. 512]), manifolds with a non-trivial action of $S U(2)$ or $S O(3)$ [38], or Toda brackets [7];

(2) surgery techniques for "propagating" positive scalar curvature from one manifold to another.

Here (1) is self-explanatory, but only covers a rather small number of examples, built out of standard building blocks such as spheres, projective spaces, and lens spaces, using fairly standard constructions. Such techniques only work on "highly symmetric" manifolds, and so one needs a way to get from these to more general manifolds. That is what is provided by the surgery method (item (2) above).

The basic result on which everything is based is the following:

Theorem 1.15 (Surgery Theorem of Gromov-Lawson [23], Schoen-Yau [61]). Let $M^{\prime}$ be a closed manifold of positive scalar curvature, not necessarily connected, and suppose $M$ is a manifold that can be obtained from $M^{\prime}$ by surgery in codimension $\geq 3$. Then $M$ also admits a metric of positive scalar curvature.

While this may not seem like much, this Surgery Theorem, together with the method of proof of the $s$-cobordism theorem, implies a reduction of the question of what manifolds $M^{n}$ admit metrics of positive scalar curvature, provided that $n \geq 5$, to bordism theory. Further application of techniques developed in [67], together with additional ideas of Jung based on the Baas theory of "bordism with singularities," ultimately reduce one down to the following statement. For simplicity, we have ignored the "twisted case," which is much more complicated to describe, though the ideas are roughly the same.

THEOREM 1.16 (Jung and Stolz). Let $M^{n}$ be a closed connected oriented manifold with fundamental group $\pi$ and dimension $n \geq 5$. Let $B \pi$ be the 
classifying space of $\pi$, or in other words, a $K(\pi, 1)$ space, which is well defined up to homotopy equivalence, and let $u: M \rightarrow B \pi$ be a classifying map for the universal cover of $\pi$ (so that the universal cover of $M$ is the pull-back under $u$ of the universal principal $\pi$-bundle).

(1) If $M$ is spin, let $[M]_{k o} \in k o_{*}(M)$ denote the ko-fundamental class of $M$ determined by the spin structure. Suppose $u_{*}\left([M]_{k o}\right) \in$ $k o_{n}(B \pi)^{+}$, the subset of $k_{n}(B \pi)$ consisting of classes $f_{*}\left([N]_{k o}\right)$ with $N^{n}$ a spin manifold of positive scalar curvature and with $f: N$ $\rightarrow B \pi$.

(2) If $w_{2}(\widetilde{M}) \neq 0$, i.e., the universal cover of $M$ is not spin, suppose $u_{*}([M]) \in H_{n}(B \pi)^{+}$, the subset of $H_{n}(B \pi)$ consisting of classes $f_{*}([N])$ with $N^{n}$ an oriented manifold of positive scalar curvature and with $f: N \rightarrow B \pi$.

Then (in either case) $M$ admits a metric of positive scalar curvature.

REMARK 1.17. Note there is a certain asymmetry between $M$ and $N$ in the theorem. While $M$ has to be connected, and while $u$ has to be a classifying map for the universal cover of $M, N$ need not be connected, and $f$ can be arbitrary. In addition, in case $(2)$, while we require $w_{2}(\widetilde{M}) \neq 0$, there is no such condition on $N$.

Another curious fact is that while, a priori, $k o_{n}(B \pi)^{+}$and $H_{n}(B \pi)^{+}$are just sets, they are in fact subgroups of $k o_{n}(B \pi)$ and $H_{n}(B \pi)$. The reason is that addition is represented in bordism theories by disjoint union of manifolds, and the disjoint union of manifolds of positive scalar curvature clearly has a metric of positive scalar curvature. Similarly, multiplication by -1 is represented by reversal of orientation or spin structure, which has no effect on the positive scalar curvature condition.

Still another case of interest is the one where $M$ is not orientable, but still $w_{2}(\widetilde{M}) \neq 0$. In this case, there is an analogue of case (2) of Theorem 1.16, but one needs to replace usual homology by homology with local coefficients. (See for example [7, Theorems 2.5 and 2.7(3)].)

1.3. Classification conjectures. In this subsection, we will discuss what answers might be expected to the question of what closed manifolds should admit metrics of positive scalar curvature, and what is known about the status of these conjectures.

First of all, for simply connected manifolds of dimension $\geq 5$, the problem is fully understood.

Theorem 1.18 (Gromov-Lawson [23], Stolz [67]). Let $M^{n}$ be a connected, simply connected closed manifold, with $n \geq 5$. Then if $w_{2}(M) \neq 0$, $M$ admits a metric of positive scalar curvature. If $w_{2}(M)=0$, so that $M$ admits a spin structure, then $M$ admits a metric of positive scalar curvature if and only if $\alpha(M)=0$ in $K O_{n}(\mathrm{pt})$. 
Dimension 2 is of course also fully understood, and we would understand dimension 3 if the Thurston Geometrization Conjecture is true. (Of course, the Poincaré Conjecture alone would settle the simply connected case.) But because of Counterexample 1.13, the situation has to be more complicated in dimension 4 . The best we might hope for would be:

Conjecture 1.19. Let $M^{4}$ be a closed simply connected 4-manifold. Then $M$ admits a metric of positive scalar curvature unless either $M$ is spin with $\widehat{A}(M) \neq 0$ or $b_{2}^{+}(M) \geq 1$ and some Seiberg-Witten invariant of $M$ is non-zero.

At the moment, we have no methods at all for attacking Conjecture 1.19. The most mysterious case of all may be the one where $b_{2}^{+}(M)=1$ and $b_{2}^{-}(M)$ is large, in which case the Seiberg-Witten invariants are not independent of the choice of metric.

In dimension 4, one possibility is to simplify the problem by allowing connected sums with $S^{2} \times S^{2}$. (By a famous argument of Wall [71], this is known to make simply connected surgery theory work in the smooth category, whereas without stabilization, smooth surgery theory fails miserably [36].) Then one obtains a rather simple result.

THEOREM 1.20. Let $M^{n}$ be a connected and simply connected smooth 4-manifold. Then $M \# k\left(S^{2} \times S^{2}\right)$ admits a metric of positive scalar curvature for some $k$ if and only if either $w_{2}(M) \neq 0$, or else $w_{2}(M)=0$ and $\widehat{A}(M)=0$.

Proof. If $M$ is spin and $\widehat{A}(M) \neq 0$, then these conditions are preserved under taking connected sums with $S^{2} \times S^{2}$. Hence, by Lichnerowicz's Theorem (Theorem 1.1), $M \# k\left(S^{2} \times S^{2}\right)$ does not admit a metric of positive scalar curvature, for any value of $k$. If $M$ is spin with $\widehat{A}(M)=0$, then the signature of $M$ vanishes (since in dimension 4, the signature and the $\widehat{A}$ genus are proportional to one another), and by Wall [71], $M \# k\left(S^{2} \times S^{2}\right)$ is diffeomorphic to a connected sum of copies of $S^{2} \times S^{2}$, for sufficiently large $k$, and thus for such $k, M \# k\left(S^{2} \times S^{2}\right)$ admits a metric of positive scalar curvature by the Surgery Theorem, Theorem 1.15. Finally, if $M$ is non-spin, then again by Wall [71] $M \# k\left(S^{2} \times S^{2}\right)$ is diffeomorphic to a connected sum of copies of $\mathbb{C P}^{2}$ and $\overline{\mathbb{C P}^{2}}$ once $k$ is sufficiently large, and so once again, $M \# k\left(S^{2} \times S^{2}\right)$ admits a metric of positive scalar curvature by the Surgery Theorem.

In dimensions $\geq 5$, various attempts have been made to extrapolate from Theorem 1.18 and Theorem 1.2 to a reasonable guess about necessary and sufficient conditions for positive scalar curvature. The best known conjectures are the following: 
Conjecture 1.21 ("Gromov-Lawson Conjecture"). Let $M^{n}$ be a closed, connected $n$-manifold with $n \geq 5$ and with fundamental group $\pi$ and classifying map $u: M \rightarrow B \pi$. If $w_{2}(\widetilde{M}) \neq 0$, then $M$ admits a metric of positive scalar curvature. If $w_{2}(M)=0$ (so we can choose a spin structure on $M$ ), then $M$ admits a metric of positive scalar curvature if and only if $\alpha_{B \pi}(M)=0$ in $K O_{n}(B \pi)$.

Conjecture 1.22 ("Gromov-Lawson-Rosenberg Conjecture"). Let $M^{n}$ be a closed, connected $n$-manifold with $n \geq 5$ and with fundamental group $\pi$ and classifying map $u: M \rightarrow B \pi$. If $w_{2}(\widetilde{\widetilde{M}}) \neq 0$, then $M$ admits a metric of positive scalar curvature. If $w_{2}(M)=0$ (so we can choose a spin structure on $M$ ), then $M$ admits a metric of positive scalar curvature if and only if $A \circ \alpha_{B \pi}(M)=0$ in $K O_{n}\left(C_{\mathbb{R}}^{*}(\pi)\right)$.

Conjecture 1.23 ("Stable Gromov-Lawson-Rosenberg Conjecture"). Let $J^{8}$ be a simply connected spin 8-manifold with $\widehat{A}(J)=1$. (This implies that $\alpha(J)$ is a "geometric representative" for Bott periodicity. For example, we can take $J$ to be a "Joyce manifold" with exceptional holonomy Spin(7).) Say that a closed $n$-manifold $M^{n}$ stably admits a metric of positive scalar curvature if $M \times J \times \cdots \times J$ admits a metric of positive scalar curvature for a sufficiently large number of $J$-factors. Then if $M$ is oriented with $w_{2}(\widetilde{M}) \neq 0, M$ always stably admits a metric of positive scalar curvature, and if $M$ is spin, then $M$ stably admits a metric of positive scalar curvature if and only if $A \circ \alpha_{B \pi}(M)=0$ in $K O_{n}\left(C_{\mathbb{R}}^{*}(\pi)\right)$.

A few words about the history are in order here. Conjecture 1.21 was hinted at in $[\mathbf{2 4}]$, but in the same paper, it was observed that the conjecture cannot always be right in the non-spin case, because of the minimal hypersurface method. (For example, apply Theorem 1.9 to the case $X=T^{n}$, $n \leq 8$. One sees that $H_{n}(X, \mathbb{Z})^{+}=0$, since otherwise, Theorem 1.9 shows $H_{n-1}(X, \mathbb{Z})^{+} \neq 0$. Iterating the construction, one eventually comes down to the case $n \leq 2$, where we know this is false. It follows that Conjecture 1.21 fails for $\left(\mathbb{C P}^{2} \times S^{2}\right) \# T^{6}$ or for $\mathbb{C P}^{4} \# T^{8}$, since these are oriented manifolds with non-spin universal cover mapping to non-trivial homology classes in $T^{6}$, resp., $T^{8}$.)

Conjecture 1.22 was proposed in [55], but only when the fundamental group $\pi$ is finite. Counterexample 1.14 shows that it fails in dimension 4 , but the conjecture was only intended to apply in dimensions 5 and up. It was shown in [60] that the conjecture fails when $\pi$ is a product of a free abelian group and a finite group, because one can use Theorem 1.9 to reduce to a low-dimensional case. A more subtle counterexample was found in [13]; here it is shown that there is a torsion-free group $\pi$ for which the assembly map $A$ is injective, but yet one can construct a manifold $M^{n}$ with fundamental group $\pi$ for which $\alpha_{B \pi}(M)=0$ in $K O_{n}(B \pi)$ and yet $M$ does not admit a metric of positive scalar curvature. What goes wrong is related to the fact 
that the periodization map per: $k o_{*}(B \pi) \rightarrow K O_{*}(B \pi)$ has a big kernel. On the other hand, if $\pi$ is such that $A$ and per are injective for $\pi$, which is the case for a large number of "nice" groups, then Conjectures 1.21 and 1.22 both hold for $\pi$ in the spin case, by a combination of Theorem 1.2 and Theorem 1.16.

Conjecture 1.23 was proposed in [57], where it was observed that the case $w_{2}(\widetilde{M}) \neq 0$ is trivial since $J$ is oriented bordant to a non-spin manifold of positive scalar curvature. In this same paper, Conjecture 1.23 was proved (or at least the proof was sketched) when $M$ is spin and $\pi$ is finite, and also when $\pi$ is torsion-free and the assembly map $A$ is injective. A much more general result was sketched in $[68]$ and $[69, \S 3]$. Namely, if the Baum-Connes Conjecture holds for $\pi$, or even if the Baum-Connes assembly map $K O_{*}^{\pi}(\underline{E \pi}) \rightarrow K O_{*}\left(C_{\mathbb{R}}^{*}(\pi)\right)$ is injective, then Conjecture 1.23 holds for $\pi$. (Here $\underline{E \pi}$ is the universal proper $\pi$-space; it coincides with $E \pi$, and $K O_{*}^{\pi}(\underline{E \pi})$ coincides with $K O_{*}(B \pi)$, provided that $\pi$ is torsion-free.)

The current status of the positive scalar curvature problem is thus very complicated. There are good reasons to believe that the Stable Conjecture, Conjecture 1.23, holds in general, but this still begs the question of what is true unstably.

For finite groups, there is a bit more one can say. First of all, it was shown in [4] that Conjecture 1.22 does hold for finite groups $\pi$ with periodic cohomology. (These are exactly the finite groups whose Sylow subgroups are all either cyclic or generalized quaternion.) By a combination of results of [30] and [8], Conjecture 1.22 in the case of non-spin universal cover also holds for elementary abelian 2-groups. Furthermore, by a combination of the results of [7] and [8], Conjecture 1.22 also holds (in both the spin and non-spin cases) for elementary abelian $p$-groups with $p$ odd, once $n$ (the dimension of the manifold) exceeds the rank of $\pi$, or more generally, provided one reformulates the conjecture just for "atoral" classes. But even in the elementary abelian case, there is one tricky case nobody has been able to handle. Namely, suppose $p$ is an odd prime and one looks at the homology class in $H_{n}\left((B \mathbb{Z} / p)^{n}\right)$ represented by $T^{n}$ in the obvious way (the map on classifying spaces induced by "reduction $\bmod p$ " $\left.\mathbb{Z}^{n} \rightarrow(\mathbb{Z} / p)^{n}\right)$. Is this homology class represented by an oriented (or spin) manifold with positive scalar curvature? As for general finite groups, nothing we know excludes the possibility that Conjecture 1.22 holds for all finite $\pi$, but in the other hand there is no obvious reason why a conjecture that fails for infinite groups should hold for finite ones.

Another "stable" conjecture which has the advantage over Conjecture 1.23 of having a simpler statement is the following:

Conjecture 1.24 (" $S^{1}$-Stability"). Let $M^{n}$ be a closed, connected $n$-manifold. Then $M$ admits a metric of positive scalar curvature if and only if $M \times S^{1}$ does. 
REMARK 1.25. One direction of this is trivial; certainly if $M$ has a metric of positive scalar curvature, then the obvious product metric on $M \times S^{1}$ has the same property. The converse would follow from most "reasonable" criteria (with good functoriality in the fundamental group) proposed for positive scalar curvature, such as Conjecture 1.21 or Conjecture 1.22 (in dimensions $\geq 5$ ). This conjecture is also compatible with Theorem 1.9. Unfortunately, Conjecture 1.24 fails in dimension 4 . To see this, choose any smooth complex hypersurface $V$ of odd degree $\geq 5$ in $\mathbb{C P}^{3}$. Then (since the degree of $V$ is odd) $V$ is a non-spin smooth simply connected 4-manifold with a Kähler structure and with $b_{2}^{+}>1$, hence by Theorem 1.12, $V$ does not admit a metric of positive scalar curvature. On the other hand, $V \times S^{1}$ is a closed oriented 5-manifold with fundamental group $\pi=\mathbb{Z}$, representing 0 in $H_{5}(B \mathbb{Z}, \mathbb{Z})=H_{5}\left(S^{1}, \mathbb{Z}\right)=0$, so $V \times S^{1}$ has a metric of positive scalar curvature by Theorem 1.16.

The author does not know of any counterexamples to Conjecture 1.24 with $n \geq 5$. Of course the big problem with this conjecture is that it doesn't settle the positive scalar curvature problem for any manifold; it simply states the equivalence of the problem on one manifold with the problem on another.

\section{The moduli space problem: what does the space of positive scalar curvature metrics look like?}

In this section, we consider the following problem. If $M^{n}$ is a closed manifold which admits at least one Riemannian metric of positive scalar curvature, what is the topology of the space $\mathfrak{R}^{+}(M)$ of all such metrics on $M$ ? In particular, is this space connected?

In general, the answer to this problem is not known, but a methodology exists for approaching it, and there are lots of partial results. The one case that is totally understood is the one where $n=2$. By Gauss-Bonnet, the only closed 2-manifolds admitting metrics of positive scalar curvature are $S^{2}$ and $\mathbb{R} \mathbb{P}^{2}$. And we have:

Theorem 2.1 ([58, Theorem 3.4]). The spaces $\mathfrak{R}^{+}\left(S^{2}\right)$ and $\mathfrak{R}^{+}\left(\mathbb{R} \mathbb{P}^{2}\right)$ are contractible.

To deal with higher-dimensional manifolds, we first need some definitions.

Definition 2.2. Suppose $M$ is a closed manifold, and let $\mathfrak{R}^{+}(M)$ denote the space of all Riemannian metrics of positive scalar curvature on $M$, with the $C^{\infty}$ topology. We assume this space is non-empty. Then two metrics $g_{0}$ and $g_{1}$ in $\mathfrak{R}^{+}(M)$ are called concordant if there is a smooth metric $g$ of positive scalar curvature on $M \times[0, a]$, for some $a>0$, which restricts in a neighborhood of 0 to the product metric $g_{0} \times \sigma$, and in a neighborhood of $a$ to the product metric $g_{1} \times \sigma$, where $\sigma$ is the standard metric on $\mathbb{R}$ 
(corresponding to $d t^{2}$ ). The metrics $g_{0}$ and $g_{1}$ are called isotopic if they lie in the same connected component (or path component, it doesn't matter) of $\mathfrak{R}^{+}(M)$.

It is easy to show that isotopic metrics of positive scalar curvature are concordant. (The original argument is in [23, Lemma 3]; see also [58, Proposition 3.3].) The converse is not at all obvious and is now known to be false (see Theorem 2.10 below), since a metric of positive scalar curvature on $M \times[0, a]$ may not necessarily be a product metric, and there is no obvious way to "straighten it." Thus $\pi_{0}\left(\mathfrak{R}^{+}(M)\right)$, the set of path components of $\mathfrak{R}^{+}(M)$, surjects onto the set $\widetilde{\pi}_{0}\left(\mathfrak{R}^{+}(M)\right)$ of concordance classes, and if the latter has more than one element (respectively, is infinite), then so is the set of path components of $\mathfrak{R}^{+}(M)$. The major result in high dimensions (specialized to the case of closed manifolds, as there is also a version for manifolds with boundary) is:

TheOREM 2.3 (Stolz [68, Theorem 3.9], [58]). Let $M^{n}$ be a connected closed spin $n$-manifold with fundamental group $\pi$ admitting a metric of positive scalar curvature, and suppose $n \geq 5$. Then there is a group $R_{n+1}(\pi)$ acting simply transitively on $\widetilde{\pi}_{0}\left(\mathfrak{R}^{+}(M)\right)$. (Thus in some sense the latter only depends on $\pi$ and on $n$.) Furthermore, there is an "index homomorphism" $\theta: R_{n+1}(\pi) \rightarrow K O_{n+1}\left(C_{\mathbb{R}}^{*}(\pi)\right)$.

Suppose furthermore that $N^{n+1}$ is a spin manifold with boundary $\partial N=$ $M$. Then a given metric of positive scalar curvature $g$ on $M$ extends to a metric of positive scalar curvature on $N$ which is a product metric in a collar neighborhood of the boundary if and only if an obstruction defined by $(N, g)$ vanishes in $R_{n+1}\left(\pi_{1}(N)\right)$.

Conjecture 2.4 (Stolz $[58])$.

The index map $\theta: R_{n+1}(\pi) \rightarrow K O_{n+1}\left(C_{\mathbb{R}}^{*}(\pi)\right)$ is "stably" an isomorphism. The notion of stability here is similar to that in Conjecture 1.23; we replace $R_{n+1}(\pi)$ by $\lim _{n+1+8 j}(\pi)$, where the maps in the inductive limit come from products with the manifold $J^{8}$.

There is also a version of Theorem 2.3 and of Conjecture 2.4 for non-spin manifolds. In this case, $R_{n+1}(\pi)$ should be replaced by $R_{n+1}(\gamma)$ with $\gamma$ as in Definition 1.4, and the real group $C^{*}$-algebra should be replaced by $C^{*}(\gamma)$.

The index map $\theta$ of Theorem 2.3 can sometimes be used to distinguish different connected components in $\mathfrak{R}^{+}(M)$. This use of the index map is quite similar to, and presumably generalizes" ${ }^{2}$, earlier uses of the "relative index" or the relative $\eta$-invariant to distinguish different connected components in $\mathfrak{R}^{+}(M)$.

For example, Hitchin [29, Theorem 4.7] proved that if $M^{n}$ is a closed spin manifold admitting a metric of positive scalar curvature, then $\pi_{0}\left(\Re^{+}(M)\right) \neq$

\footnotetext{
${ }^{2}$ The author is not sure if all the details of proving that the two constructions coincide have been verified, but there is reason to believe this shouldn't be so hard.
} 
0 provided that $n \equiv 0$ or $1 \bmod 8$, and $\pi_{1}\left(\mathfrak{R}^{+}(M)\right) \neq 0$ provided that $n \equiv-1$ or $0 \bmod 8$. Examination of his proof shows that in terms of the language of Theorem 2.3, he was really showing that the composite

$$
R_{n+1}(\pi) \stackrel{\ominus}{\rightarrow} K O_{n+1}\left(C_{\mathbb{R}}^{*}(\pi)\right) \rightarrow K O_{n+1}(\mathrm{pt}) \cong \mathbb{Z} / 2
$$

is surjective when $n+1 \equiv 1$ or $2 \bmod 8$, basically because we know there are exotic spheres in dimensions 1 or $2 \bmod 8$ for which the $\alpha$-invariant is non-zero.

Since there are still no high-dimensional manifolds for which the topology of $\mathfrak{R}^{+}(M)$ is fully understood, the rest of this section will consist largely of a catalog of examples.

For example, one of the earliest results on the topology of $\mathfrak{R}^{+}(M)$ is the following:

Theorem 2.5 (Gromov-Lawson [24, Theorem 4.47]). The space $\mathfrak{R}^{+}\left(S^{7}\right)$ has infinitely many connected components; in fact, $\widetilde{\pi}_{0}\left(\mathfrak{R}^{+}\left(S^{7}\right)\right)$ is infinite.

Proof Following [24]. The idea is to construct "exotic metrics" on $S^{7}$ by using the fact that there are many ways to write $S^{7}$ as the unit sphere bundle of an oriented $\mathbb{R}^{4}$-bundle $E$ over $S^{4}$. Such bundles are classified by two integer invariants: the first Pontrjagin class $p_{1}$ (always an even number) and the Euler class $e$. The unit sphere bundle $S(E)=\Sigma^{7}$ (with respect to some choice of smooth metric on the vector bundle) is (oriented) homotopy equivalent to $S^{7}$ if $e=1$, and as shown by Milnor [44], $p_{1}^{2} \equiv 4(\bmod 7)$ is necessary for $\Sigma$ to be diffeomorphic to $S^{7}$. The value $p_{1}=2$ corresponds to the usual presentation of $S^{7}$ as the unit sphere bundle of a quaternionic line bundle over $\mathbb{H} \mathbb{P}^{1} \cong S^{4}$. But in [35], it is shown that the $h$-cobordism classes of smooth homotopy 7 -spheres constitute a cyclic group $\Theta_{7}$ of order 28 , and thus there are other values of $p_{1}\left(\right.$ such as $\left.p_{1}=2+28=30\right)$ for which $S(E)$ is diffeomorphic to $S^{7}$.

Now it is easy to construct a metric of positive scalar curvature on the unit disk bundle $D(E)$ of $E$ which is a product metric in a collar neighborhood of the boundary $S(E) \cong S^{7}$. Suppose the metric obtained this way on $S^{7}$ were "standard," i.e., concordant to the standard spherical metric $g_{0}$. Then we could take a metric on $D^{8}$ of positive scalar curvature which is a product metric $g_{0} \times \sigma$ (see Definition 2.2 for the notation) in a collar neighborhood of the boundary $S^{7}$, and glue the two metrics together (after first inserting a "fitting," a copy of $S^{7} \times I$ with a concordance metric) to get a metric of positive scalar curvature on $M^{8}=D(E) \cup_{S(E)}\left(S^{7} \times I\right) \cup_{S^{7}} D^{8}$. This is a contradiction, since $M$ is a spin manifold with $\widehat{A}(M) \neq 0$. In fact, a slight variant of this calculation shows that if one takes two different values for $p_{1}(E)$ (but for both of which we have $S(E) \cong S^{7}$ ), then the metrics obtained on $S^{7}$ cannot be concordant. Thus since there are infinitely many values of $p_{1}$ for which $S(E) \cong S^{7}$, there are infinitely many concordance classes of positive scalar curvature metrics on $S^{7}$. 
Now let's give a different construction for exotic positive scalar curvature metrics on homotopy 7-spheres, which works in any dimension $4 k-1, k \geq 2$. Afterwards, we will say a bit about the special case $k=1$. We actually do not know if $\mathfrak{R}^{+}\left(S^{3}\right)$ has infinitely many connected components or not.

THEOREM 2.6. The space $\mathfrak{R}^{+}\left(S^{4 k-1}\right)$ has infinitely many connected components for $k \geq 2$; in fact, the index invariant $R_{4 k} \rightarrow K O_{4 k} \cong \mathbb{Z}$ is non-trivial.

Proof. Let $M^{4 k}$ be the parallelizable manifold with boundary obtained from the $E_{8}$ plumbing as in [45]. In more specific terms, $M$ is obtained by starting with the $4 k$-disk $D^{4 k}$ and adding on $82 k$-handles $D^{2 k} \times D^{2 k}$, plumbed together to intersect according to the Cartan matrix of $E_{8}$. Note that $M$ may be viewed as the result of doing surgery on 8 copies of $S^{2 k-1}$ embedded into the boundary $S^{4 k-1}$ of $D^{4 k}$. Since we are assuming that $k \geq 2$, the codimension condition of the Surgery Theorem is satisfied, and so $M$ admits a metric of positive scalar curvature extending a standard "round" metric on the original $4 k$-disk $D^{4 k}$, and a product metric on the boundary $\Sigma^{4 k-1}=\partial M$. (Strictly speaking, we need the version of the Surgery Theorem that applies to manifolds with boundary. This is a variant on Theorem 1.15 due to Gajer. See [17] and [68, Theorem 3.3] for details.) As argued by Kervaire and Milnor, $M$ is parallelizable and $\Sigma$ is a nonstandard homotopy sphere. To quickly sum up the argument, the main points are these:

(1) $M$ and $\Sigma$ are simply connected, since they are the result of highly connected surgeries. (N.B.: The assumption $k \geq 2$ is used here; if $k=1, \Sigma$ turns out to be the Poincaré homology sphere, the quotient of $S^{3}$ by the binary icosahedral group of order 120, and so is not simply connected. We will come back to this point later.)

(2) $M$ is parallelizable, since it is built by framed surgery from the tangent bundles of spheres.

(3) $\Sigma$ is a homology sphere, because of the fact that the Cartan matrix of $E_{8}$ is unimodular. (This part is still valid even when $k=1$.)

(4) Consider $N^{4 k}=M^{4 k} \cup_{\Sigma} \operatorname{cone}(\Sigma)$. This is a topological $4 k$-manifold. However, it cannot be smooth, and so $\Sigma$ is not diffeomorphic to $S^{4 k-1}$. The reason is the following. Suppose we had $\Sigma \cong S^{4 k-1}$, cone $(\Sigma) \cong D^{4 k}$. Then $N$ would be smooth and almost parallelizable (parallelizable off a disk). Thus all its Pontrjagin classes would vanish except for $p_{k}$ in degree $4 k$. (Any lower Pontrjagin class would be detectable by its restriction to a proper skeleton, and thus by its restriction to $M^{4 k}$. But $M^{4 k}$ is parallelizable.) However, the signature of $N$ is 8 , since by construction, its intersection form on middle homology is given by $E_{8}$, which is unimodular of rank 8 . Thus, by the Hirzebruch signature formula, the term in the $L$-class of $M$ involving $p_{k}$ must evaluate to 8 . This is a contradiction, since $\left\langle p_{k},[M]\right\rangle$ is integral and we know the coefficient $\mu_{k}$ of $p_{k}$ in $L$; it's 
a complicated rational number related to the Bernoulli numbers:

$$
\mu_{k}=\frac{2^{2 k}\left(2^{2 k-1}-1\right)}{(2 k) !} B_{k}
$$

$[\mathbf{2 8}, \S 1.5]$. In particular, its numerator is such that $\left\langle\mu_{k} p_{k},[M]\right\rangle$ can't be 8 .

Now let's go back to the issue of positive scalar curvature metrics. Recall we've constructed using surgery a metric of positive scalar curvature on $M^{4 k}$ which restricts to a product metric in a collar neighborhood of the boundary homotopy sphere $\Sigma^{4 k-1}$. By [35], there is a finite number $m$ such that the $m$-fold connected sum $\overbrace{\Sigma \# \Sigma \# \cdots \# \Sigma}^{m}$ is diffeomorphic to a standard sphere $S^{4 k-1}$. Thus there is a (spin) cobordism $P^{4 k}$, the trace of a surgery on a union on $S^{0}$ 's, from $\coprod_{j=1}^{m} \Sigma^{4 k-1}$ to $\#_{j=1}^{m} \Sigma^{4 k-1} \cong S^{4 k-1}$. Again by the Surgery Theorem, there is a metric of positive scalar curvature on $P^{4 k}$ which is a product metric on a neighborhood of each boundary component: the metric constructed above (coming from $M^{4 k}$ ) on each copy of $\Sigma^{4 k-1}$, and some metric of positive scalar curvature $g$ on $S^{4 k-1}$. We claim that $g$ is not in the same concordance class as the standard round metric $g_{0}$ on $S^{4 k-1}$, and in fact that the index obstruction to extending $g$ to a metric on $D^{4 k}$, restricting to a product metric near the boundary, is non-zero. This will prove the theorem.

Indeed, if our claim is false, there is a metric of positive scalar curvature on

$$
Q^{4 k}=\left(\coprod_{j=1}^{m} M^{4 k}\right) \cup_{\coprod_{j=1}^{m} \Sigma^{4 k-1}} P \cup_{S^{4 k-1}} D^{4 k}
$$

extending the metrics we've constructed on each copy of $M$ and on $P$. (See Figure 1.)

Now $Q^{4 k}$ is a closed spin manifold, and by an argument similar to the one used above with $N^{4 k}$, it is almost parallelizable. So all its Pontrjagin classes vanish except for $p_{k}$ in top degree. Furthermore, the construction shows that the intersection form of $Q$ on middle homology is a direct sum of $m$ copies of $E_{8}$, so the signature of $Q^{4 k}$ is $8 m$. That tells us, as above, that $\mu_{k}\left\langle p_{k},[M]\right\rangle=$ $8 m$. But the coefficient of $p_{k}$ in the $\widehat{A}$ polynomial, $-\left(B_{n} / 2(2 n) !\right)$, is also nonzero. So $\widehat{A}(Q) \neq 0$, contradicting Theorem 1.1. This completes the proof, since we've shown that the index obstruction to extending $g$ over a disk $D^{4 k}$ is non-zero.

In [24] and [58], the question of whether $\mathfrak{R}^{+}\left(S^{3}\right)$ is disconnected was left open. On the one hand, might expect $\pi_{0}\left(\Re^{+}\left(S^{3}\right)\right)$ to be infinite via a certain index calculation, but on the other hand, Hitchin showed in $[\mathbf{2 9}, \S 3]$ that the space of left invariant positive scalar curvature metrics on $S U(2) \cong S^{3}$ is contractible. He also computed the $\eta$-invariants for these metrics, and showed that it varies continuously. The method of proof used above seems 


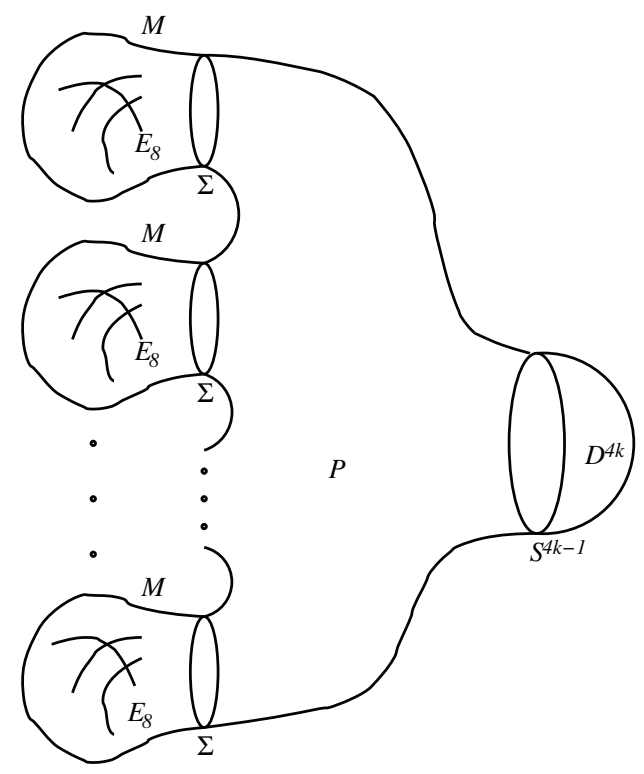

Figure 1. The construction of the manifold $Q^{4 k}$.

at first to be promising, in terms of showing that the $E_{8}$ manifold $M^{4}$ has a metric of positive scalar curvature restricting to a product metric on a neighborhood of the boundary, the Poincaré homology sphere $\Sigma^{3}$. If this were the case, there would be some hope of lifting the metric on $\Sigma^{3}$ to the covering space $S^{3}$, and showing that the metric constructed this way on $S^{3}$ is "exotic," say by means of an $\eta$-invariant calculation. But unfortunately, the argument breaks down right at the first step, because the codimension condition in the Surgery Theorem 1.15 isn't satisfied.

Next, we discuss various methods for detecting non-triviality of the topology of $\mathfrak{R}^{+}(M), M$ odd-dimensional, via eta invariants. Unfortunately, the ordinary (untwisted) eta invariant of the Dirac operator doesn't help much, since it vanishes identically for metrics of positive scalar curvature on spin manifolds in dimension $\equiv 1 \bmod 4$ (see for example [20, Lemma 1.7.10]), while in dimension $\equiv 3 \bmod 4$, it only helps if one can control the $\widehat{\mathcal{A}}$ term in the Atiyah-Patodi-Singer Theorem [1]. However, there is also a reasonably large literature using the twisted eta invariant (for the Dirac operator twisted by a flat bundle, and especially for the formal difference of two such twists of the same dimension) to prove facts about $\mathfrak{R}^{+}(M)$ when $M$ is not simply connected. We will just give a few representative examples, and leave it to the reader to consult $[\mathbf{5}],[\mathbf{3 9}],[\mathbf{1 9}],[\mathbf{1 8}],[\mathbf{2 9}]$, and $[\mathbf{6}]$ for more results and details.

THEOREM 2.7. Let $M$ be a closed connected spin manifold of odd dimension, and let $g_{0}$ and $g_{1}$ be metrics of positive scalar curvature on $M$. Let $\rho$ be 
a virtual unitary representation of $\pi=\pi_{1}(M)$ of virtual dimension 0 , i.e., a formal difference of two finite dimensional unitary representations $\rho^{+}$and $\rho^{-}$of $\pi$ with $\operatorname{dim} \rho^{+}=\operatorname{dim} \rho^{-}$. Let $\eta_{0}(\rho)=\eta\left(\not D_{0}, \rho, 0\right), \eta_{1}(\rho)=\eta\left(\not D_{1}, \rho, 0\right)$, where $\not_{0}$ and $\not_{1}$ are the Dirac operators for the metrics $g_{0}$ and $g_{1}$, and $\eta\left(\not D_{j}, \rho\right)$ is defined by

$$
\eta\left(\not D_{j}, \rho\right)=\eta\left(\not D_{j} \otimes 1_{\mathcal{V}_{\rho^{+}}}\right)-\eta\left(\not D_{j} \otimes 1_{\mathcal{V}_{\rho^{+}}}\right)
$$

$\mathcal{V}_{\rho^{ \pm}}$denoting the flat vector bundle defined by $\rho^{ \pm}$. Then if $\eta_{0}(\rho) \neq \eta_{1}(\rho)$, $g_{0}$ and $g_{1}$ and not concordant, and in particular, do not lie in the same connected component of $\mathfrak{R}^{+}(M)$.

PROOF. Suppose $g_{0}$ and $g_{1}$ are concordant. Then there is a metric $g$ of positive scalar curvature on $M \times[0,1]$, restricting to a product of $g_{0}$ with a metric on the line in a collar neighborhood of $M \times\{0\}$ (which we may identify with $M$ ) and to a product of $g_{1}$ with a metric on the line in a collar neighborhood of $M \times\{1\}$ (which we may identify with $-M, M$ with the orientation reversed). The representations $\rho^{ \pm}$of $\pi$ give vector bundles $\mathcal{V}_{\rho^{ \pm}}$ on $M \times[0,1]$, which we equip with flat connections. Now consider the index problem for $\not D_{M \times[0,1]} \otimes 1_{\mathcal{V}_{\rho^{ \pm}}}$with Atiyah-Patodi-Singer boundary conditions on $M \times\{0\}$ and $M \times\{1\}$. Since $M \times[0,1]$ has positive scalar curvature and the vector bundle is flat, the Lichnerowicz identity (1.1) shows the kernel of $\not D_{M \times[0,1]}$ vanishes, and thus the index is 0 . Furthermore, since $\mathcal{V}_{\rho^{ \pm}}$is flat, its Chern character reduces by Chern-Weil theory simply to $\operatorname{dim} \rho^{ \pm}$. So by the Atiyah-Patodi-Singer Theorem [1], we have

$$
0=\frac{1}{2}\left(\eta\left(\not D_{1}, \rho^{ \pm}\right)-\eta\left(\not D_{0}, \rho^{ \pm}\right)\right)+\int_{M \times[0,1]} \widehat{\mathcal{A}} \cdot \operatorname{dim} \rho^{ \pm}
$$

Subtract equation $(2.1)$ for $\rho^{-}$from equation $(2.1)$ for $\rho^{+}$, and the $\widehat{\mathcal{A}}$ terms cancel. So if $\eta_{0}(\rho) \neq \eta_{1}(\rho)$, we get a contradiction, and the result follows.

Corollary 2.8 (Botvinnik and Gilkey [5, Theorem 0.2]). Let $M$ be a closed connected spin $(4 k+1)$-manifold with finite fundamental group $\pi$, admitting a metric of positive scalar curvature, and assume that $\pi$ has a non-zero virtual unitary representation $\rho$ of virtual dimension 0 , satisfying the parity condition $\operatorname{Tr} \rho(h)=-\operatorname{Tr} \rho\left(h^{-1}\right)$ for all $h \in \pi$. Then $\widetilde{\pi}_{0}\left(\mathfrak{R}^{+}(M)\right)$ is infinite.

SKETCH OF PROOF. An induction argument reduces everything to the case where $\pi \cong \mathbb{Z} / p$ is cyclic. We will give the proof in the case $p$ is odd, which results in a slight simplification since, in this case, any non-trivial irreducible representation $\sigma: \pi \rightarrow U(1)$ gives a non-zero $\rho$ of virtual dimension 0 satisfying the parity condition (namely $\rho=\sigma-\bar{\sigma}$ ), and any lens space with fundamental group $\pi$ is automatically spin. Then because of Theorem 2.3, it suffices to prove the result when $M$ itself is a 5-dimensional 
lens space $L^{5}$. (To pass to the case $k>1$, simply take a product with copies of a Kummer surface and/or a Joyce manifold $J^{8}$, using the fact that $\eta(M \times N, \rho)=\eta(M, \rho) \widehat{A}(N)$ when $N$ has dimension divisible by $4[\mathbf{2 0}$, Lemma 1.7.18].) By the Atiyah-Hirzebruch spectral sequence for bordism, $\left|\Omega_{5}^{\text {spin }}(\mathbb{Z} / p)\right|=p^{2}$ when $p$ is odd. But the eta invariants of lens spaces for the standard metric of constant curvature, $\eta\left(L^{n}\right)(\rho)$, are computed in [12] (for the signature operator, but the same method also works for the Dirac operator) and in [20, Theorem 1.8.5]. To fix notation, let $L^{5}(\tau)=S^{5} / \tau$ be a lens space of dimension $n$ with fundamental group $\pi=\mathbb{Z} / p$, associated to the representation $\tau=\left(\lambda, \lambda^{a_{1}}, \lambda^{a_{2}}\right)$ of $\pi$, where $\lambda$ sends the generator of $\pi$ to $e^{2 \pi i / p}$ and $1 \leq a_{1}, a_{2} \leq p-1$.) Note that $\tau$ acts freely on $\mathbb{C}^{3} \backslash\{0\}$, and thus freely on the unit sphere $S^{5}$. The formula in [20, Theorem 1.8.5] gives

$$
\eta\left(L^{5}(\tau)\right)(\rho)=\frac{1}{p} \sum_{h \in \pi, h \neq 1} \operatorname{Tr} \rho(h) \operatorname{det}(\tau(h))^{1 / 2} \operatorname{det}(\tau(h)-1)^{-1} .
$$

Because of the parity condition on $\rho$, the quantity inside the summation sign is invariant under replacing $h$ by $h^{-1}$. (In fact that's why we need the parity condition, for if $\operatorname{Tr}(\rho(h))=\operatorname{Tr}\left(\rho\left(h^{-1}\right)\right)$ for all $h$, then the quantity being summed is odd and the $\eta$-invariant vanishes.) We might as well take $\rho=\lambda^{1 / 2}-\lambda^{-1 / 2}$. Then if $\tau=(\lambda, \lambda, \lambda),(2.2)$ becomes

$$
\begin{aligned}
\eta\left(L^{5}(\lambda, \lambda, \lambda)\right)\left(\lambda^{1 / 2}-\lambda^{-1 / 2}\right) & =\frac{1}{p} \sum_{h \in \pi, h \neq 1} \frac{1}{\left(\lambda(h)^{1 / 2}-\lambda(h)^{-1 / 2}\right)^{2}} \\
& =\frac{1}{p} \sum_{j=1}^{p-1} \frac{1}{4 \sin ^{2}(2 \pi j / p)}=\mu>0
\end{aligned}
$$

while if $\tau=\left(\lambda, \lambda, \lambda^{-1}\right),(2.2)$ becomes

$$
\begin{aligned}
& \eta\left(L^{5}\left(\lambda, \lambda, \lambda^{-1}\right)\right)\left(\lambda^{1 / 2}-\lambda^{-1 / 2}\right) \\
& =\frac{1}{p} \sum_{h \in \pi, h \neq 1} \frac{1}{\left(\lambda(h)^{1 / 2}-\lambda(h)^{-1 / 2}\right)\left(\lambda(h)^{-1 / 2}-\lambda(h)^{1 / 2}\right)} \\
& =\frac{1}{p} \sum_{j=1}^{p-1} \frac{1}{-4 \sin ^{2}(2 \pi j / p)}=-\mu<0 .
\end{aligned}
$$

Let $M^{5}=L^{5}(\lambda, \lambda, \lambda)$ and call $g_{0}$ the standard metric of positive scalar curvature on $M$. Then $\eta\left(g_{0}\right)(\rho)=\mu>0$. But since $\left|\Omega_{5}^{\text {spin }}(\mathbb{Z} / p)\right|<\infty$, there is a spin bordism (over $B \mathbb{Z} / p$ ) from a disjoint union of finitely many copies of $L^{5}\left(\lambda, \lambda, \lambda^{-1}\right)$, say $r$ copies, to $M$. We can use this (as in the proof of Theorem 2.6) to push the standard metric on this disjoint union over to a metric $g_{1}$ on $M$, which by an argument similar to that in the proof of Theorem 2.7 must satisfy $\eta\left(g_{1}\right)(\rho)=-r \mu<0$. Since we just showed the 
standard metric $g_{0}$ on $M$ satisfies $\eta\left(g_{1}\right)(\rho)>0$, Theorem 2.7 shows $g_{0}$ and $g_{1}$ are not concordant. Furthermore, we can construct metrics on $M$ with infinitely many values of the $\eta$-invariant, since we are free to replace $r$ by $r+p^{2} j$ for any $j$. (Recall $\left|\Omega_{5}^{\text {spin }}(\mathbb{Z} / p)\right|=p^{2}$.) This completes the proof for this case. The other cases are similar.

REMARK 2.9. In the language of Theorem 2.3, Corollary 2.8 says that under these hypotheses, $R_{4 k+2}(\pi)$ is infinite. Another way to prove this would be to show directly that the index invariant

$$
\theta: R_{4 k+2}(\pi) \rightarrow K O_{4 k+2}\left(C_{\mathbb{R}}^{*}(\pi)\right)=K O_{4 k+2}(\mathbb{R} \pi)
$$

has infinite image. The representation-theoretic hypothesis guarantees that $\pi$ has at least one irreducible representation of complex type, i.e., that $\mathbb{R} \pi$ has at least one summand of the form $M_{m}(\mathbb{C})$. This summand contributes a $\mathbb{Z}$ to $K O_{4 k+2}(\mathbb{R} \pi)$, and Corollary 2.8 says $\theta$ hits this $\mathbb{Z}$ non-trivially. This lends a bit of support to the surjectivity part of Conjecture 2.4.

All of the results so far, showing that the topology of $\mathfrak{R}^{+}(M)$ is nontrivial, have been based on index theory or the eta-invariant. In dimension 4, Seiberg-Witten theory can also be used, leading to a remarkable result:

TheOREM 2.10 (Ruberman [59]). There is a simply connected 4-manifold with infinitely many concordant but nonisotopic metrics of positive scalar curvature.

I am not aware of any results like this in higher dimensions.

Finally, there are some other results that imply something interesting about the space $\mathfrak{R}^{+}(M)$. For example, in [10], it is shown that if $g$ is a metric on $M$ and $(M, g)$ admits a spin cover with nonzero parallel spinors, then $g$ cannot be deformed to a metric of positive scalar curvature. If $M$ is spin and simply connected, and has nonzero parallel spinors for the metric $g$, then there can be no metrics of positive scalar curvature in a neighborhood of $g$.

\section{Complete metrics of positive scalar curvature on noncompact manifolds}

The study of complete metrics of positive scalar curvature on noncompact manifolds is noticeably more complicated than for closed manifolds, and in this section we will just touch on a few of the issues involved. We have divided the discussion into two subsections: one on global results and one on metrics within a fixed quasi-isometry class.

3.1. Global results. Some noncompact manifolds do not admit any complete metrics of positive scalar curvature at all; others admit such metrics, but not if the scalar curvature is bounded below by a positive constant. 
While we still don't know what manifolds belong in these classes, we do have the following conjecture.

Conjecture 3.1 ([56, Conjecture 7.1$])$. Let $X^{n}$ be a closed manifold which does not admit a metric of positive scalar curvature. Then

(1) $X^{n} \times \mathbb{R}$ does not admit a complete metric of positive scalar curvature;

(2) $X^{n} \times \mathbb{R}^{2}$ admits no complete metrics of uniformly positive scalar curvature.

This conjecture is known to be true [24, $\S 6$ and $\S 7]$ if $n \leq 2$, i.e., if $X$ is a point, $S^{1}, T^{2}$, a Klein bottle, or a compact surface without boundary with a hyperbolic metric. The conjecture cannot be strengthened any further because of:

Proposition 3.2 ([56, Proposition 7.2]). Let $X^{n}$ be any closed manifold. Then $X^{n} \times \mathbb{R}^{2}$ admits complete metrics of (non-uniformly) positive scalar curvature, and $X^{n} \times \mathbb{R}^{k}$ admits complete metrics of uniformly positive scalar curvature when $k \geq 3$.

Additional positive evidence for something like Conjecture 3.1 comes from noncompact index theory, at least in the case of spin manifolds. For example, one has:

Theorem 3.3 ([24, Corollary $\left.\mathrm{B}_{2}\right]$ - see also $\left.[\mathbf{5 0}]\right)$. Let $X^{n}$ be a closed spin manifold with $\widehat{A}(X) \neq 0$. Then $X^{n} \times \mathbb{R}$ does not admit a complete metric of positive scalar curvature.

The following is based on some of the same ideas, but carried out in the context of more sophisticated Kasparov theory.

THEOREM 3.4. Let $X^{n}$ be a closed spin manifold, and assume that $A\left(\alpha_{B \pi}(X)\right) \neq 0$ in $K O_{n}\left(C_{\mathbb{R}}^{*}(\pi)\right)$. Then $X^{n} \times \mathbb{R}$ does not admit a complete metric of uniformly positive scalar curvature.

Proof. As some of the details are a bit complicated, we prefer to begin by explaining first how the proof works when $\pi$ is trivial, i.e., when we replace $\widehat{A}(X)$ in Theorem 3.3 by $\alpha(X) \in K O_{n}$ (which can be non-zero also in dimensions 1 and $2 \bmod 8$ ). Then we will explain how to modify the proof to cover the general case.

Fix a Riemannian metric on $X$, and let $D_{X}$ be the $C \ell_{n}$-linear Dirac operator on $X$, as used in the proof of Theorem 1.1. This operator defines a class $\left[\not D_{X}\right]$ in the Kasparov group $K K O\left(C^{\mathbb{R}}(X), C \ell_{n}\right)$, and $\alpha(X)$ is the image of this class in $K K O\left(\mathbb{R}, C \ell_{n}\right)=K K O\left(C^{\mathbb{R}}(\mathrm{pt}), C \ell_{n}\right)$ under the map of Kasparov groups induced by the inclusion $\mathbb{R} \hookrightarrow C^{\mathbb{R}}(X)$, or dually, the "collapse map" $c: X \rightarrow$ pt. 
Next, observe that we have a similar class $\left[\not D_{X \times \mathbb{R}}\right]$, defined by the Dirac operator on $X \times \mathbb{R}$ for the product metric on this manifold, in the Kasparov group $K K O\left(C_{0}^{\mathbb{R}}(X \times \mathbb{R}), C \ell_{n+1}\right)$. This class is just the external Kasparov product of $\left[D_{X}\right]$ with the Dirac operator class on the line. We have a commutative diagram of groups:

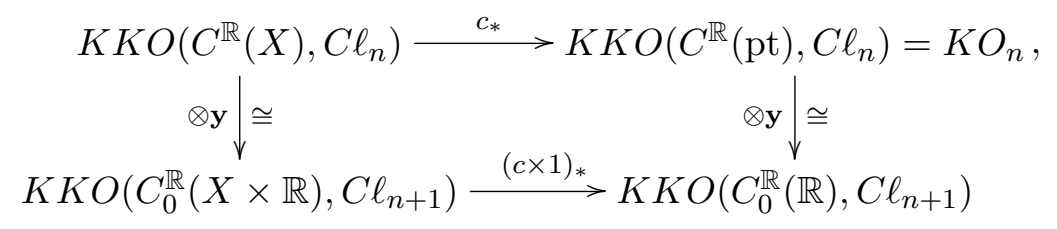

where $\mathbf{y} \in K K O\left(C_{0}^{\mathbb{R}}(\mathbb{R}), C \ell_{1}\right)$ is $\not D_{\mathbb{R}}$ or the Bott periodicity operator (see $[\mathbf{2}, \S 19.2])$, and $\otimes \mathbf{y}$ denotes the (external) Kasparov product. This gives rise to the commutative diagram of Kasparov elements:

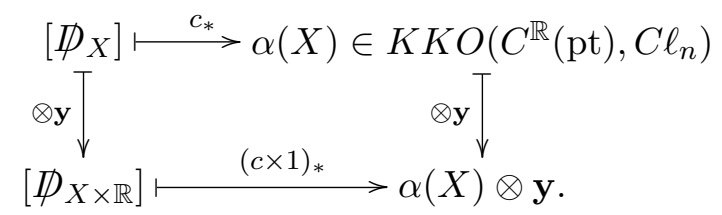

Now suppose that $X \times \mathbb{R}$ admits a complete metric of uniformly positive scalar curvature, say $g$. Then the Dirac operator $\not D_{g}$ for this metric $g$, which is essentially self-adjoint since $g$ is complete, and the Dirac operator for the product metric on $X \times \mathbb{R}$, define the same Kasparov class $\left[\not D_{g}\right]=\left[\not D_{X \times \mathbb{R}}\right]$. (This point is made in [27]; the essential fact is that we are dealing with a Kasparov class for the algebra of continuous functions which vanish at infinity, in which continuous functions of compact support are dense, and any two complete metrics on a noncompact manifold, when restricted to a fixed compact set, are homotopic through homotopies of complete metrics supported on a slightly larger compact set.)

Next we note that Bott periodicity implies that the Kasparov class $\mathbf{y}$, which lies in $K K O\left(C_{0}^{\mathbb{R}}(\mathbb{R}), C \ell_{1}\right)$, has an inverse $\mathbf{x} \in K K O\left(C \ell_{1}, C_{0}^{\mathbb{R}}(\mathbb{R})\right) \cong$ $K O^{-1}(\mathbb{R})$ (see $\left.[\mathbf{2}, \S 19.2]\right)$. Thus, putting this and the insensitivity of the Dirac class on $X \times \mathbb{R}$ to the choice of complete metric together with (3.1), we obtain the equality

$$
\alpha(X)=\mathbf{x} \otimes_{C_{0}^{\mathbb{R}}(\mathbb{R})}(c \times 1)_{*}\left(\left[\not D_{g}\right]\right) \in K K O\left(C \ell_{1}, C \ell_{n+1}\right) \cong K O_{n}
$$

The rest of the proof consists of showing that this Kasparov product vanishes, using the fact that $g$ has uniformly positive scalar curvature. For this, we need explicit realizations for the classes $\mathbf{x}$ and $(c \times 1)_{*}\left(\left[\not D_{g}\right]\right)$, as well as the Kasparov calculus for computing the product. Note incidentally that $C \ell_{1}=\mathbb{R}+\mathbb{R} i$, where $i^{2}=-1$, so we can identify $C \ell_{1}$ with $\mathbb{C}$, the grading given by complex conjugation. The class $\mathbf{x}$ is represented by the $C \ell_{1}-C_{0}^{\mathbb{R}}(\mathbb{R})$ bimodule $C \ell_{1} \otimes C_{0}^{\mathbb{R}}(\mathbb{R})=C_{0}^{\mathbb{R}}(\mathbb{R})+i C_{0}^{\mathbb{R}}(\mathbb{R})$, together with the operator $F$ 
given by multiplication by

$$
F=\left(\begin{array}{cc}
0 & -i f(x) \\
i f(x) & 0
\end{array}\right)
$$

where $f$ is a continuous function on the line that tends to 1 at $+\infty$ and to -1 at $-\infty$. It will be convenient to assume that $-1 \leq f \leq 1$, that $f$ is smooth, and that $f \equiv-1$ on $(-\infty,-a], f \equiv 1$ on $[a, \infty)$, for some $a>0$. Thus $F^{2} \equiv 1$ except on $[-a, a]$. The class $(c \times 1)_{*}\left(\left[\not D_{g}\right]\right)$ is represented by the graded real Hilbert space $\mathcal{H}$ of $L^{2}$ sections of the $C \ell_{n+1}$-linear spinor bundle on $(X \times \mathbb{R}, g)$, the operator $D=\not D_{g}\left(\not D_{g}^{2}\right)^{-1}$, and the obvious action of $C_{0}^{\mathbb{R}}(\mathbb{R})$ by multiplication operators. (The fact that $g$ has uniformly positive scalar curvature implies that the differential operator $\not_{g}^{2}$ is bounded away from 0 , hence invertible, so we can use the above formula for $D$ instead of the more usual $\not D_{g}\left(1+\not D_{g}^{2}\right)^{-1}$. In particular, our choice of $D$ satisfies $D^{2}=1$ precisely, not just "approximately.")

We proceed to the compute the Kasparov product in (3.2). It acts on the graded Hilbert space $C_{0}^{\mathbb{C}}(\mathbb{R}) \widehat{\otimes}_{C_{0}^{\mathbb{R}}(\mathbb{R})} \mathcal{H}=C \ell_{1} \widehat{\otimes} \mathcal{H}=\mathcal{H}_{\mathbb{C}}$, with the obvious action of $C \ell_{1}$ on the left, and the issue is to compute the relevant Fredholm operator $G=F \# D$.

From the recipe for the Kasparov product (see [2, $§ 18.4]$ ), the operator $G$ should be chosen to be of the form

$$
G=M^{1 / 4} F M^{1 / 4}+N^{1 / 4} D N^{1 / 4}
$$

where $0 \leq M, N \leq 1, M+N=1$, so that $G^{2}-1$ is compact and the anticommutator $\{G, F\}$ is positive modulo compacts. (We are letting $F$ act on function spaces on $X^{n} \times \mathbb{R}$ in the obvious way, through strictly speaking we should write $F \widehat{\otimes} 1$, etc.) We have

$$
\begin{aligned}
G^{2}-1= & M^{1 / 4} F M^{1 / 2} F M^{1 / 4}+N^{1 / 4} D N^{1 / 2} D N^{1 / 4}-1 \\
& +\left\{M^{1 / 4} F M^{1 / 4}, N^{1 / 4} D N^{1 / 4}\right\}
\end{aligned}
$$

In our situation, $D^{2}=1$, and $\{F, D\}$ is basically $[D, i f]$, which is the commutator of two pseudodifferential operators of order 0 , hence is pseudodifferential of negative order, but may not be compact since we are on a noncompact manifold. This suggests taking $N$ to be a multiplication operator given by a nonnegative function on $\mathbb{R}$ of compact support, with $F^{2}=1$ on the support of $M$, in which case $M$ will commute with $F$ and

$$
\begin{aligned}
& M^{1 / 4} F M^{1 / 2} F M^{1 / 4}+N^{1 / 4} D N^{1 / 2} D N^{1 / 4}-1 \\
& =M F^{2}+N^{1 / 2} D^{2} N^{1 / 2}-1+N^{1 / 4}\left(D N^{1 / 2} D-N^{1 / 4} D^{2} N^{1 / 4}\right) N^{1 / 4} \\
& \equiv M F^{2}+N^{1 / 2} D^{2} N^{1 / 2}-1=M+N-1=0
\end{aligned}
$$


modulo compacts. On the other hand,

$$
\begin{aligned}
& \left\{M^{1 / 4} F M^{1 / 4}, N^{1 / 4} D N^{1 / 4}\right\} \\
& =N^{1 / 4}\left\{M^{1 / 2} F, D\right\} N^{1 / 4}
\end{aligned}
$$

is compact since $\left\{M^{1 / 2} F, D\right\}$ is pseudodifferential of negative order and $N$ has compact support. So $G^{2}-1$ is compact, and by a similar calculation, $\left\{N^{1 / 4} D N^{1 / 4}, F\right\}$ is compact, hence $\{G, F\}$ is positive modulo compacts. Thus $G$ as we've written it down is a representative for the "sharp product" $F \# D$, and so by $(3.2), \alpha(X)$ can be computed from the finite-dimensional kernel of $G$, which is a graded $C \ell_{1}-C \ell_{n+1}$ bimodule. Next, observe that we obtain a homotopy of Kasparov $C \ell_{1}-C \ell_{n+1}$ bimodules by letting the support of $N$ grow and letting the support of $M$ shrink, so that $M$ tends strongly to 1 and in the limit, the operator $G$ becomes simply $D$. Since $D^{2}=1$, ker $D=0$. This means the Kasparov module is trivial, i.e., $\alpha(X)=0$.

Now we indicate how to extend the proof to the case of an arbitrary group $\pi$. As in the proof of Theorem 1.2 , let $\mathcal{V}_{B \pi}$ be the "universal flat bundle" over $B \pi$ with fibers that are rank-one free (right) modules over $C_{\mathbb{R}}^{*}(\pi)$. Pull this bundle back to a bundle $\mathcal{V}_{X}$ over $X$ via $f: X \rightarrow B \pi$, and extend the bundle in the obvious way to a bundle $\mathcal{V}$ over $X \times \mathbb{R}$. We now repeat the whole argument, replacing $\not D_{g}$ by $\not_{g} \otimes 1$ acting on the $C \ell_{n+1}$-module spinor bundle with coefficients in $\mathcal{V}$. We construct the operator $D$ as before, this time obtaining a class $\left[D_{X \times \mathbb{R}, \mathcal{V}}\right] \in K K O\left(C_{0}^{\mathbb{R}}(X \times \mathbb{R}), C_{\mathbb{R}}^{*}(\pi) \widehat{\otimes} C \ell_{n+1}\right)$ mapping to $A\left(\alpha_{B \pi}(X)\right) \otimes \mathbf{y}$ in $K K O\left(C_{0}^{\mathbb{R}}(\mathbb{R}), C_{\mathbb{R}}^{*}(\pi) \widehat{\otimes} C \ell_{n+1}\right)$ under $(c \otimes 1)_{*}$. As before we take the Kasparov product with the class $\mathbf{x}$ and obtain the desired conclusion.

Corollary 3.5. Conjecture 1.22 implies part (1) of Conjecture 3.1, if we weaken positive scalar curvature to uniformly positive scalar curvature, at least in the spin case with $n \geq 5$.

Proof. Conjecture 1.22 is simply the statement that the hypothesis of Theorem 3.4 is equivalent to $X^{n}$ not having a metric of positive scalar curvature.

REMARK 3.6. We should mention that any counterexample to Conjecture 1.24 is also a counterexample to Conjecture 3.1. Indeed, suppose $X^{n}$ is a closed manifold that does not admit a metric of positive scalar curvature, but such that $X^{n} \times S^{1}$ does admit such a metric. Then the lift of this metric to the covering space $X^{n} \times \mathbb{R}$ has uniformly positive scalar curvature, contradicting both parts of Conjecture 3.1. Thus the example mentioned in Remark 1.25 above (which incidentally was simply connected but not spin) shows that Conjecture 3.1 fails if $n=4$, even if $X$ is simply connected. One can construct a similar example (again with $n=4$ ) with $X$ spin by 
using part (1) of Counterexample 1.13. However, we know of no counterexamples to Conjecture 3.1 with $n>4$, and Corollary 3.5 suggests that such counterexamples will be difficult to find.

In dimension 3, somewhat more is known; for example we have:

Theorem 3.7 (Schoen and Yau [63, Theorem 4]). Let $M$ be $a$ 3-dimensional connected manifold admitting a complete metric of positive scalar curvature. Then $\pi_{1}(M)$ cannot contain a subgroup isomorphic to the fundamental group of a closed Riemann surface of positive genus.

Aside from products of compact manifolds with Euclidean spaces, another very interesting general class of noncompact manifolds comes from locally symmetric spaces. The following was proved by Block and Weinberger:

TheOREM 3.8 (Block and Weinberger [3]). Let $M=\Gamma \backslash G / K$ be an irreducible locally symmetric space of noncompact type and finite volume. Then $M$ can be given a complete metric of uniformly positive scalar curvature if and only if $\Gamma$ is an arithmetic lattice of $\mathbb{Q}$-rank $\geq 3$.

Note incidentally that $\Gamma$ is cocompact, i.e., $M$ is compact, if and only if $\Gamma$ has $\mathbb{Q}$-rank 0. This case is included in the theorem, but if $M$ is compact, it cannot have a metric of positive scalar curvature because of Theorems 1.2 and 1.7, or other similar results. Also, we are not assuming a priori that $\Gamma$ is arithmetic, though if it is not, Theorem 3.8 says that $M$ never has a complete metric of uniformly positive scalar curvature.

3.2. Metrics in a fixed quasi-isometry class. Many of the interesting results on positive scalar curvature for noncompact manifolds involve specifying the quasi-isometry class of the metric, or what is almost the same, specifying the rate of growth or decay of the metric at infinity.

One of the most effective tools for producing results of this sort is the coarse index theory of Roe, as outlined in [51], [52], and [53]. The basic construction involves a $C^{*}$-algebra $C^{*}(M)$ attached to a "coarse space," a metric space in which closed bounded sets are compact. For present purposes we should really work with the real version of the construction and write $C_{\mathbb{R}}^{*}(M)$, but we will suppress the $\mathbb{R}$ for notational convenience. The algebra $C^{*}(M)$ is the completion of the locally compact, finite propagation operators on $M$, acting on an auxiliary separable Hilbert space, and when $M$ is a complete Riemannian manifold, it really only depends on the quasiisometry class of the metric. For example, when $M$ is compact, $C^{*}(M)$ is just the algebra $\mathcal{K}$ of compact operators (which is Morita equivalent to the scalars). Then we have the following result, generalizing Theorem 1.1 to the noncompact case.

Theorem 3.9 (See [52, Definition 3.7 and Proposition 3.8].). Let $\left(M^{n}, g\right)$ be a complete Riemannian spin manifold. Then the Dirac operator for the 
metric $g$ and the given spin structure defines a class ind $\not_{g} \in K O_{n}\left(C^{*}(M)\right)$, and if this class is non-zero, $g$ cannot have uniformly positive scalar curvature. In fact, when the index is non-zero, there can be no complete metric of uniformly positive scalar curvature in the same quasi-isometry class.

Proof. Roe states and proves this in the complex case, so we will just indicate how to obtain the refinement in real $K$-theory. As usual, we work with the $C \ell_{n}$-linear Dirac operator $D_{g}$. By (1.1), the spectrum of this operator is bounded away from 0 if $g$ has uniformly positive scalar curvature. As in the proof of Theorem 3.4, choose a continuous real-valued (odd) function $f$ on $\mathbb{R}$ with $-1 \leq f \leq 1$ and with $f(x) \rightarrow 1$ as $x \rightarrow+\infty, f(x) \rightarrow-1$ as $x \rightarrow$ $-\infty$, and observe that $f\left(D_{g}\right)$ is $C \ell_{n}$-linear, odd (with respect to the grading of the spinor bundle) and bounded. Furthermore, since the hyperbolic equation $u_{t}=i \not D_{g} u$ has finite propagation speed, $f\left(\not D_{g}\right)$ is a multiplier of $C^{*}(M)$, and defines a class ind $\not D_{g} \in K K O\left(\mathbb{R}, C^{*}(M) \widehat{\otimes} C \ell_{n}\right) \cong K O_{n}\left(C^{*}(M)\right)$, and ind $\not_{g}$ is evidently 0 if $\not_{g}$ has a bounded inverse, which is the case if $g$ has uniformly positive scalar curvature. Furthermore, this index class ind $\not_{g}$ is invariant under homotopies of the metric within the same quasi-isometry class (since such homotopies give homotopies of the Kasparov class), so if the index is $\neq 0$, there can be no complete metric of uniformly positive scalar curvature in the same quasi-isometry class.

Most of the known results about non-existence of complete metrics of uniformly positive scalar curvature in quasi-isometry classes of noncompact manifolds come from applying various tricks to detect the index class ind $\not_{g}$ topologically. Of course, since it is the coarse geometry of $M$, not its usual topology, that is relevant here, "topologically" means "in terms of coarse invariants," such as coarse $K O$-homology $K O X_{*}(M)$ in the sense of [52]. There is a coarse assembly map $K_{O} O X_{*}(M) \rightarrow K O_{*}\left(C^{*}(M)\right)$ defined in [52, Ch. 8]. When $M$ is uniformly contractible, this is simply the map that takes the class $[D]$ of an elliptic operator $D$ to ind $(D)$ as defined above. More generally, this map is defined by taking indices of the images of $D$ on "coarse approximations" to $X$.

Conjecture 3.10 (Coarse Baum-Connes Conjecture [52, Conjecture 8.2]). For any proper metric space $M$ of bounded geometry, the coarse assembly map $K_{O} O X_{*}(M) \rightarrow K O_{*}\left(C^{*}(M)\right)$ is an isomorphism.

Incidentally, a counterexample to the surjectivity part of Conjecture 3.10 is known $[\mathbf{2 6}, \S 6]$, but we shall only need the injectivity part. Putting together Theorem 3.9 and Conjecture 3.10, we obtain:

Proposition 3.11 (Roe). If $M$ is a uniformly contractible complete Riemannian manifold of bounded geometry, and if (the injectivity part of the) 
Conjecture 3.10 holds for $M$, then there is no complete metric of uniformly positive scalar curvature in the quasi-isometry class of the given metric on $M$.

Proof. In this case, the coarse assembly map takes $\left[\not D_{g}\right] \in K O_{n}(M)$, which is a generator in $K O_{n}(M) \cong K O_{n}\left(\mathbb{R}^{n}\right) \cong \mathbb{Z}$, to ind $\not_{g}$, which by Theorem 3.9 is an obstruction to uniformly positive scalar curvature in the quasi-isometry class of the given metric on $M$.

In some cases, one can prove Conjecture 3.10 and apply this result. For example, we have the following results:

Theorem 3.12 (Yu [74, Corollary 7.3]). Let $M$ be a uniformly contractible complete Riemannian manifold with finite asymptotic dimension. Then $M$ cannot have uniformly positive scalar curvature.

Theorem $3.13(\mathrm{Yu}[\mathbf{7 5}$, Corollary 1.3]). Let $M$ be a complete Riemannian manifold with bounded geometry. If $M$ is uniformly contractible and admits a uniform embedding into Hilbert space, then $M$ cannot have uniformly positive scalar curvature.

Theorem 3.14 (Gong and Yu [21, Corollary 4.3]). Let $M$ be a uniformly contractible complete Riemannian manifold with bounded geometry. If $M$ has subexponential volume growth, then $M$ cannot have uniformly positive scalar curvature.

Another result related to Theorem 3.8 is the following:

Theorem 3.15 (Chang [9]). Let $M=\Gamma \backslash G / K$ be an irreducible locally symmetric space of noncompact type and finite volume, and suppose $\Gamma$ is an arithmetic lattice of $\mathbb{Q}$-rank $\geq 3$ (so that by Theorem 3.8, $M$ admits a metric of positive scalar curvature). Then no metric of positive scalar curvature on $M$ can be quasi-isometric to the standard locally symmetric metric.

Finally, there are results on positive scalar curvature in a quasi-isometry class that involve still other versions of noncompact index theory. A typical example is:

TheOREM 3.16 (Whyte [72]). Assume that $M^{n}$ is a complete connected spin manifold with bounded curvature and uniformly positive scalar curvature, and that $N^{n}$ is a closed spin manifold with $\widehat{A}(N)>0$. Let $S$ be a discrete subset of $M$. Then the connected sum of $M$ with one copy of $N$ attached at each point of $S$ (see Figure 2) admits a complete metric of uniformly positive curvature (in the canonical quasi-isometry class of metrics) if $[S]=0$ in $H_{0}^{u f}(M)$, the uniformly finite homology of $M$, and does not admit any complete metric of positive scalar curvature if $[S] \neq 0$ in $H_{0}^{u f}(M)$. 


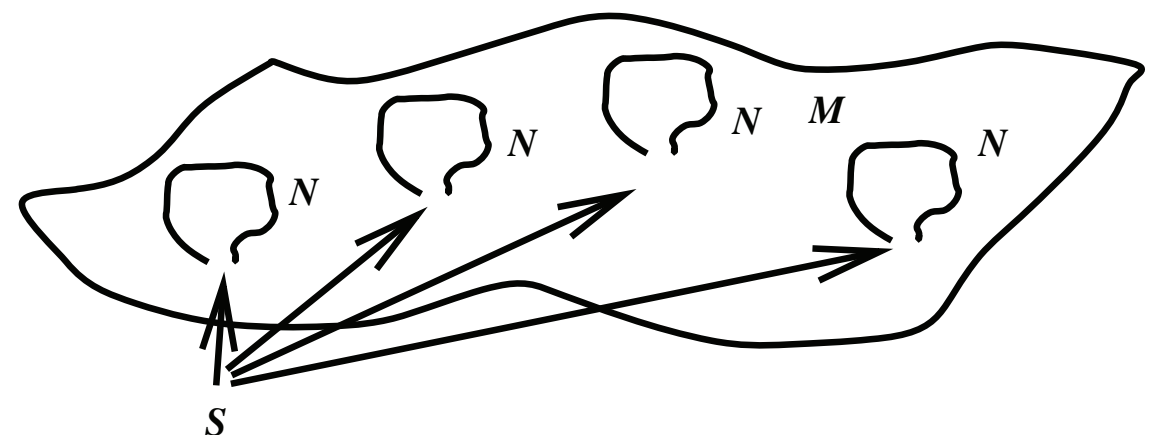

FiguRE 2. The connected sum along a discrete subset.

\section{Miscellaneous topics}

4.1. The second Kazdan-Warner class. Recall from Theorem 0.1 that if $M^{n}$ is a closed manifold of dimension $n \geq 3$, and if $M$ admits a metric with nonnegative scalar curvature but not one with positive scalar curvature, then any such metric must be Ricci-flat. Futaki [16] and Dessai [11] have obtained additional restrictions on such manifolds. For example, $[\mathbf{1 6}]$ shows that simply connected manifolds of dimension $>4$ in class $(2)$ of Theorem 0.1 must be spin, have non-vanishing $\widehat{A}$-genus, and have exceptional holonomy $S U(2 m), S p(n)$ or $\operatorname{Spin}(7)$. Furthermore, Futaki obtains additional constraints on the $\widehat{A}$-genus, and Dessai shows that the first Pontrjagin class must be non-trivial.

One also has certain results that constrain "almost non-negative scalar curvature." A closed manifold $M$ is said [14] to have "almost non-negative scalar curvature" if, for any $\varepsilon>0$, there is a Riemannian metric $g$ with sectional curvature $\leq 1$ and with scalar curvature $\kappa$ and diameter $d$ satisfying $\kappa \geq-\varepsilon / d^{2}$. The results of $[\mathbf{1 4}]$ say that in some cases, this is impossible unless $M$ lies in the second Kazdan-Warner class.

4.2. Metrics with negative scalar curvature. Lohkamp has also shown that the results of Section 2 are also really very special to positive scalar curvature. On any closed manifold $M^{n}$ with $n \geq 3$, Lohkamp showed [41] that the space of metrics of negative scalar curvature is contractible, with a retraction onto the subspace of metrics of constant scalar curvature -1 . Furthermore, an arbitrary metric can be perturbed so as to decrease its scalar curvature on a prescribed open set, without changing the overall "shape" of the manifold. More precisely, one has:

THEOREM 4.1 (Lohkamp [42]). Let $\left(M^{n}, g\right)$ be a Riemannian n-manifold with $n>2$ and with scalar curvature function $\kappa$. Let $U$ be an open subset of $M$, and let $f$ be a smooth function on $M$ such that $f<\kappa$ on $U$ and $f=\kappa$ on $M \backslash U$. Then for each positive $\varepsilon$, there is a smooth metric $g_{\epsilon}$ on 
$M$ such that $g_{\epsilon}=g$ outside the $\varepsilon$-neighborhood of $U$ and such that the scalar curvature function $\kappa_{\varepsilon}$ of $g_{\epsilon}$ satisfies $f-\varepsilon \leq \kappa_{\varepsilon} \leq f$ on the $\varepsilon$-neighborhood of $U$. Moreover, $g_{\varepsilon}$ can be chosen arbitrarily close to $g$ in the $C^{0}$ topology.

\section{References}

[1] M.F. Atiyah, V.K. Patodi, and I.M. Singer, Spectral asymmetry and Riemannian geometry, I, Math. Proc. Cambridge Philos. Soc., 77 (1975), 43-69, MR0397797 (53 \#1655a).

[2] B. Blackadar, K-theory for operator algebras, second ed., Mathematical Sciences Research Institute Publications, 5, Cambridge University Press, Cambridge, 1998, MR1656031 (99g:46104).

[3] J. Block and S. Weinberger, Arithmetic manifolds of positive scalar curvature, J. Differential Geom., 52(2) (1999), 375-406, MR1758300 (2001h:53047).

[4] B. Botvinnik, P. Gilkey, and S. Stolz, The Gromov-Lawson-Rosenberg conjecture for groups with periodic cohomology, J. Differential Geom., 46(3) (1997), 374-405, MR1484887 (98i:58227).

[5] B. Botvinnik and P.B. Gilkey, The eta invariant and metrics of positive scalar curvature, Math. Ann., 302(3) (1995), 507-517, MR1339924 (96f:58159).

[6] B. Botvinnik and P.B. Gilkey, The eta invariant and the Gromov-Lawson conjecture for elementary abelian groups of odd order, Topology Appl., 80(1-2) (1997), 43-53, MR1469465 (99f:58194).

[7] B. Botvinnik and J. Rosenberg, The Yamabe invariant for non-simply connected manifolds, J. Differential Geom., 62(2) (2002), 175-208, MR1988502 (2004j:53045).

[8] B. Botvinnik and J. Rosenberg, Positive scalar curvature for manifolds with elementary abelian fundamental group, Proc. Amer. Math. Soc., 133(2) (2005), 545-556 (electronic), MR2093079 (2005g:53057).

[9] S.S. Chang, Coarse obstructions to positive scalar curvature in noncompact arithmetic manifolds, J. Differential Geom., 57(1) (2001), 1-21, MR1871489 (2002j:53037).

[10] X. Dai, X. Wang, and G. Wei, On the stability of Riemannian manifold with parallel spinors, Invent. Math., 161(1) (2005), 151-176, MR2178660.

[11] A. Dessai, On the topology of scalar-flat manifolds, Bull. London Math. Soc., 33(2) (2001), 203-209, MR1815425 (2002b:53063).

[12] H. Donnelly, Eta invariants for G-spaces, Indiana Univ. Math. J., 27(6) (1978), 889-918, MR511246 (80m:58042).

[13] W. Dwyer, T. Schick, and S. Stolz, Remarks on a conjecture of Gromov and Lawson, High-dimensional manifold topology, World Sci. Publishing, River Edge, NJ, 2003, 159-176, MR2048721 (2005f:53043).

[14] Fuquan Fang, Index of Dirac operator and scalar curvature almost non-negative manifolds, Asian J. Math., 7(1) (2003), 31-38, MR2015240 (2005a:58032).

[15] R. Fintushel and R.J. Stern, Double node neighborhoods and families of simply connected 4-manifolds with $b^{+}=1$, J. Amer. Math. Soc., 19(1) (2006), 171-180 (electronic), MR2169045.

[16] A. Futaki, Scalar-flat closed manifolds not admitting positive scalar curvature metrics, Invent. Math., 112(1) (1993), 23-29, MR1207476 (94f:53072).

[17] P. Gajer, Riemannian metrics of positive scalar curvature on compact manifolds with boundary, Ann. Global Anal. Geom., 5(3) (1987), 179-191, MR962295 (89m:53061).

[18] P.B. Gilkey, The eta invariant, manifolds of positive scalar curvature, and equivariant bordism, Geometry, topology and physics (Campinas, 1996), de Gruyter, Berlin, 1997, 157-171, MR1605220 (99k:58175).

[19] P.B. Gilkey, The eta invariant of Pin manifolds with cyclic fundamental groups, Period. Math. Hungar., 36(2-3) (1998), 139-170, MR1694601 (2001a:58034). 
[20] P.B. Gilkey, J.V. Leahy, and J. Park, Spectral geometry, Riemannian submersions, and the Gromov-Lawson conjecture, Studies in Advanced Mathematics, Chapman \& Hall/CRC, Boca Raton, FL, 1999, MR1707341 (2000j:58035).

[21] G. Gong and G. Yu, Volume growth and positive scalar curvature, Geom. Funct. Anal., 10(4) (2000), 821-828, MR1791141 (2001m:53055).

[22] A. Gray, The volume of a small geodesic ball of a Riemannian manifold, Michigan Math. J., 20 (1973), 329-344, MR0339002 (49 \#3765).

[23] M. Gromov and H.B. Lawson, Jr., The classification of simply connected manifolds of positive scalar curvature, Ann. of Math. (2), 111(3) (1980), 423-434, MR577131 (81h:53036).

[24] M. Gromov and H.B. Lawson, Jr., Positive scalar curvature and the Dirac operator on complete Riemannian manifolds, Inst. Hautes Études Sci. Publ. Math., 58 (1983), 83-196, MR720933 (85g:58082).

[25] B. Hanke, D. Kotschick, and J. Wehrheim, Dissolving four-manifolds and positive scalar curvature, Math. Z., 245(3) (2003), 545-555, MR2021570 (2005b:57059).

[26] N. Higson, V. Lafforgue, and G. Skandalis, Counterexamples to the Baum-Connes conjecture, Geom. Funct. Anal., 12(2) (2002), 330-354, MR1911663 (2003g:19007).

[27] N. Higson, K-homology and operators on non-compact manifolds, Unpublished preprint, available at http://www.math.psu.edu/higson/ResearchPapers.html, 1988.

[28] F. Hirzebruch, Topological methods in algebraic geometry, Classics in Mathematics, Springer-Verlag, Berlin, 1995, Translated from the German and Appendix One by R.L.E. Schwarzenberger, With a preface to the third English edition by the author and Schwarzenberger, Appendix Two by A. Borel, Reprint of the 1978 edition, MR1335917 (96c:57002).

[29] N. Hitchin, Harmonic spinors, Advances in Math., 14 (1974), 1-55, MR0358873 (50 \#11332).

[30] M. Joachim, Toral classes and the Gromov-Lawson-Rosenberg conjecture for elementary abelian 2-groups, Arch. Math. (Basel), 83(5) (2004), 461-466, MR2102644 (2005g:53050).

[31] M. Joachim and T. Schick, Positive and negative results concerning the GromovLawson-Rosenberg conjecture, Geometry and topology: Aarhus (1998), Contemp. Math., 258, Amer. Math. Soc., Providence, RI, 2000, 213-226, MR1778107 (2002g:53079).

[32] J.L. Kazdan and F.W. Warner, A direct approach to the determination of Gaussian and scalar curvature functions, Invent. Math., 28 (1975), 227-230, MR0375154 (51 \#11350).

[33] J.L. Kazdan and F.W. Warner, Existence and conformal deformation of metrics with prescribed Gaussian and scalar curvatures, Ann. of Math. (2), 101 (1975), 317-331, MR0375153 (51 \#11349).

[34] J.L. Kazdan and F.W. Warner, Scalar curvature and conformal deformation of Riemannian structure, J. Differential Geometry, 10 (1975), 113-134, MR0365409 (51 \#1661).

[35] M.A. Kervaire and J.W. Milnor, Groups of homotopy spheres, I, Ann. of Math. (2), 77 (1963), 504-537, MR0148075 (26 \#5584).

[36] R.C. Kirby and L.R. Taylor, A survey of 4-manifolds through the eyes of surgery, Surveys on surgery theory, Vol. 2, Ann. of Math. Stud., 149, Princeton Univ. Press, Princeton, NJ, 2001, 387-421, MR1818779 (2002a:57028).

[37] H.B. Lawson, Jr. and M.-L. Michelsohn, Spin geometry, Princeton Mathematical Series, 38, Princeton University Press, Princeton, NJ, 1989, MR1031992 (91g:53001).

[38] H.B. Lawson, Jr. and S.-T. Yau, Scalar curvature, non-abelian group actions, and the degree of symmetry of exotic spheres, Comment. Math. Helv., 49 (1974), 232-244, MR0358841 (50 \#11300). 
[39] E. Leichtnam and P. Piazza, On higher eta-invariants and metrics of positive scalar curvature, K-Theory, 24(4) (2001), 341-359, MR1885126 (2002k:58051).

[40] A. Lichnerowicz, Spineurs harmoniques, C.R. Acad. Sci. Paris, 257 (1963), 7-9, MR0156292 (27 \#6218).

[41] J. Lohkamp, The space of negative scalar curvature metrics, Invent. Math., 110(4) (1992), 403-407, MR1185590 (93h:58025).

[42] J. Lohkamp, Scalar curvature and hammocks, Math. Ann., 313(3) (1999), 385-407, MR1678604 (2000a:53059).

[43] J. Lohkamp, Positive scalar curvature in $\operatorname{dim} \geq 8$, C.R. Math. Acad. Sci. Paris, 343 (2006), no. 9, 585-588.

[44] J. Milnor, On manifolds homeomorphic to the 7-sphere, Ann. of Math. (2), 64 (1956), 399-405, MR0082103 (18,498d).

[45] J.W. Milnor and M.A. Kervaire, Bernoulli numbers, homotopy groups, and a theorem of Rohlin, Proc. Internat. Congress Math., 1958, Cambridge Univ. Press, New York, 1960, 454-458, MR0121801 (22 \#12531).

[46] A.S. Miščenko and A.T. Fomenko, The index of elliptic operators over $C^{*}$-algebras, Izv. Akad. Nauk SSSR Ser. Mat., 43(4) (1979), 831-859, 967, MR548506 (81i:46075).

[47] J.W. Morgan, The Seiberg-Witten equations and applications to the topology of smooth four-manifolds, Mathematical Notes, 44, Princeton University Press, Princeton, NJ, 1996, MR1367507 (97d:57042).

[48] J. Park, Simply connected symplectic 4-manifolds with $b_{2}^{+}=1$ and $c_{1}^{2}=2$, Invent. Math., 159(3) (2005), 657-667, MR2125736 (2006c:57024).

[49] J. Park, A.I. Stipsicz, and Z. Szabó, Exotic smooth structures on $\mathbb{C P}^{2} \# 5 \overline{\mathbb{C P}^{2}}$, Math. Res. Lett., 12(5-6) (2005), 701-712, MR2189231.

[50] J. Roe, Partitioning noncompact manifolds and the dual Toeplitz problem, Operator algebras and applications, Vol. 1, London Math. Soc. Lecture Note Ser., 135, Cambridge Univ. Press, Cambridge, 1988, 187-228, MR996446 (90i:58186).

[51] J. Roe, Coarse cohomology and index theory on complete Riemannian manifolds, Mem. Amer. Math. Soc., 104(497) (1993), MR1147350 (94a:58193).

[52] J. Roe, Index theory, coarse geometry, and topology of manifolds, CBMS Regional Conference Series in Mathematics, 90, Published for the Conference Board of the Mathematical Sciences, Washington, DC, 1996, MR1399087 (97h:58155).

[53] J. Roe, Lectures on coarse geometry, University Lecture Series, 31, American Mathematical Society, Providence, RI, 2003, MR2007488 (2004g:53050).

[54] J. Rosenberg, $C^{*}$-algebras, positive scalar curvature, and the Novikov conjecture, III, Topology, 25(3) (1986), 319-336, MR842428 (88f:58141).

[55] J. Rosenberg, The KO-assembly map and positive scalar curvature, Algebraic topology Poznań 1989, Lecture Notes in Math., 1474, Springer, Berlin, 1991, 170-182, MR1133900 (92m:53060).

[56] J. Rosenberg and S. Stolz, Manifolds of positive scalar curvature, Algebraic topology and its applications, Math. Sci. Res. Inst. Publ., 27, Springer, New York, 1994, 241-267, MR1268192.

[57] J. Rosenberg and S. Stolz, A "stable" version of the Gromov-Lawson conjecture, The Čech centennial (Boston, MA, 1993), Contemp. Math., 181, Amer. Math. Soc., Providence, RI, 1995, 405-418, MR1321004 (96m:53042).

[58] J. Rosenberg and S. Stolz, Metrics of positive scalar curvature and connections with surgery, Surveys on surgery theory, 2, Ann. of Math. Stud., 149, Princeton Univ. Press, Princeton, NJ, 2001, 353-386, MR1818778 (2002f:53054).

[59] D. Ruberman, Positive scalar curvature, diffeomorphisms and the Seiberg-Witten invariants, Geom. Topol., 5 (2001), 895-924 (electronic), MR1874146 (2002k:57076).

[60] T. Schick, A counterexample to the (unstable) Gromov-Lawson-Rosenberg conjecture, Topology, 37(6) (1998), 1165-1168, MR1632971 (99j:53049). 
[61] R. Schoen and S.-T. Yau, On the structure of manifolds with positive scalar curvature, Manuscripta Math., 28(1-3) (1979), 159-183, MR535700 (80k:53064).

[62] R. Schoen and S.-T. Yau, Existence of incompressible minimal surfaces and the topology of three-dimensional manifolds with nonnegative scalar curvature, Ann. of Math. (2), 110(1) (1979), 127-142, MR541332 (81k:58029).

[63] R. Schoen and S.-T. Yau, Complete three-dimensional manifolds with positive Ricci curvature and scalar curvature, Seminar on Differential Geometry, Ann. of Math. Stud., 102, Princeton Univ. Press, Princeton, NJ, 1982, 09-228, MR645740 (83k:53060).

[64] R. Schoen and S.-T. Yau, The structure of manifolds with positive scalar curvature, Directions in partial differential equations (Madison, WI, 1985), Publ. Math. Res. Center Univ. Wisconsin, 54, Academic Press, Boston, MA, 1987, 235-242, MR1013841 (90e:53059).

[65] N. Smale, Generic regularity of homologically area minimizing hypersurfaces in eightdimensional manifolds, Comm. Anal. Geom., 1(2) (1993), 217-228, MR1243523 (95b:49065).

[66] A.I. Stipsicz and Z. Szabó, An exotic smooth structure on $\mathbb{C P}^{2} \# 6 \overline{\mathbb{C P}^{2}}$, Geom. Topol., 9 (2005), 813-832 (electronic), MR2140993.

[67] S. Stolz, Simply connected manifolds of positive scalar curvature, Ann. of Math. (2), 136(3) (1992), 511-540, MR1189863 (93i:57033).

[68] S. Stolz, Positive scalar curvature metrics-existence and classification questions, Proceedings of the International Congress of Mathematicians, Vol. 1, 2 (Zürich, 1994) (Basel), Birkhäuser, 1995, 625-636, MR1403963 (98h:53063).

[69] S. Stolz, Manifolds of positive scalar curvature, Topology of high-dimensional manifolds, No. 1, 2 (Trieste, 2001), ICTP Lect. Notes, 9, Abdus Salam Int. Cent. Theoret. Phys., Trieste, 2002, Papers from the School on High-Dimensional Manifold Topology held in Trieste, May 21-June 8, 2001; Available electronically at http://users.ictp.it/ pub_off/lectures/, 661-709, MR1937026 (2003m:53059).

[70] C.H. Taubes, The Seiberg-Witten invariants and symplectic forms, Math. Res. Lett., 1(6) (1994), 809-822, MR1306023 (95j:57039).

[71] C.T.C. Wall, On simply-connected 4-manifolds, J. London Math. Soc., 39 (1964), 141-149, MR0163324 (29 \#627).

[72] K. Whyte, Index theory with bounded geometry, the uniformly finite $\widehat{A}$ class, and infinite connected sums, J. Differential Geom., 59(1) (2001), 1-14, MR1909246 (2003a:58031).

[73] E. Witten, Monopoles and four-manifolds, Math. Res. Lett., 1(6) (1994), 769-796, MR1306021 (96d:57035).

[74] G. Yu, The Novikov conjecture for groups with finite asymptotic dimension, Ann. of Math. (2), 147(2) (1998), 325-355, MR1626745 (99k:57072).

[75] G. Yu, The coarse Baum-Connes conjecture for spaces which admit a uniform embedding into Hilbert space, Invent. Math., 139(1) (2000), 201-240, MR1728880 (2000j:19005).

Department of Mathematics, University of Maryland,

College PARK, MD 20742, USA

E-mail address: jmr@math.umd.edu 